\title{
Dynamics of photoinduced instability in ferroelectric photorefractive crystals
}

\author{
Anna N. Morozovska*, Vyacheslav V. Obukhovsky, Vasiliy V. Lemeshko \\ Taras Shevchenko Kyiv University, Radiophysic Department, \\ 01033, Kyiv, Volodymyrs'ka str.64, Ukraine. \\ e-mail:vvo@mail.univ.kiev.ua, "morozo@iop.kiev.ua.
}

\begin{abstract}
In the paper we theoretically consider the dynamics of the inner field generated by recharging trap waves propagation and spatial-temporal features of the photoinduced light scattering caused by these phenomena in the ferroelectric photorefractive crystals under steady illumination by laser beam. The transverse instability of the interacting light beams plays an important role and both the photovoltaic and the diffusion mechanisms of carrier transfer are taken into account in the proposed theory. For the first time it has been shown that, due to the influence of transverse photovoltaic current and typical boundary conditions for inner field, among all possible scenarios of transverse instabilities the boundary circle will be realized in the perfect crystal. Due to this phenomenon the periodical, quasi-periodical or aperiodical photoinduced light scattering appear in the system, depending on the structure of growth non-homogeneities and the character of photoinduced fluctuations caused by them. The correlation between the structure of scattering light waves and the type of these fluctuations has been considered in details for ilmenites. The great attention has been paid to the description of the optical autowaves generation and dynamic halo scattering in these materials. All the main theoretical results are in a good agreement with the available experimental data.
\end{abstract}

Keywords: photorefractive crystals, recharging waves, photoinduced light scattering, optical autowaves.

Paper received 11.09.01; revised manuscript received 27.11.01; accepted for publication 12.12.01.

\section{Introduction}

Ferroelectric photorefractive crystals (PRC) are ones of the basic materials for dynamic holography and information processing. But rather often it is impossible to predict the response of the material to laser illumination. For example, various dynamic effects appears in the scattering light even under steady irradiation by laser beam of these materials: periodic pulsation (optical autowaves [1]), non-stationary maxima $[2,3]$, chaotic bursts and optical vortices [4] and so on. In this paper, we consider the dynamic halo scattering appeared in $\mathrm{LiTaO}_{3}$ doped with 0.05 wgh. $\%$ of $\mathrm{Cr}[5]$ and the generation of optical autowaves recorded in $\mathrm{LiNbO}_{3}$ doped with $0.02 \div 0.07 \mathrm{wgh} . \%$ of $\mathrm{Fe}$ [6]. Such distinctive features of these effects as rather regular spatial conic structure an impulse-like time behavior lead to the appearance of the scattering ring (hallo) on the screen perpendicular to the pump wave direction. This ring spreads out and temporally disappears. The process is periodic in the case of autowave generation.
For the adequate description of such effects in PRC it is necessary to evolve the theory in which the transverse instability of the interacting light beams plays an important role and both the photovoltaic and the diffusion mechanisms of carrier transfer are taken into account [7-11].

We used the following well-known phenomenon as the basic idea for the evolving of the theory. The nonuniform time-dependent inner photoinduced field appears in PRC even under steady laser illumination. It can be detected due to the linear electro-optic (EO) effect. The theoretical interpretation of this phenomenon based on the analyses of space and temporal dependence of the degree of completion of the crystal impurity levels (traps). Under definite conditions, the non-uniform and non-stationary traps completion proved to be energetically preferable. In this case either damping or periodic recharging trap waves can propagate in the crystal in the linear approximation [12]. The spatial structure and evolution of the scattered light is defined by this inner field in accordance with the EO effect. 


\section{A. N. Morozovska et al.: Dynamics of photoinduced instability in ferroelectric...}

In the paper, we consider the dynamic of the inner field generated by recharging trap waves propagation and spatial-temporal features of the photoinduced light scattering (PILS) caused by these phenomena.

\section{The problem}

Let us consider the dynamic photoinduced effects in PRC without an inversion center. We'll be interested in the instability generation threshold and in the form of transverse instability realization for the electric fields in PRC under steady illumination by laser beam along the polar (optical) axis.

In order to obtain relevant results, let's calculate the interaction of the laser beam with the dynamic hologram (photoinduced by a spatially non-uniform inner field) recorded by itself in the crystal within the framework of the perturbation theory.

1) At first, we shell find the inner electric field in the non-depleted pump wave approximation with definite intensity. The dynamics of inner field is determined from the system of equations for the degree of completion of donor and acceptor levels by electrons, the concentration of electrons in the conduction band, and from the Maxwell equations that can be reduced to the Poisson equation for the quasi-static electric field. Hereinafter acceptor levels are considered as rather deep and therefore completely filled by electrons, while donor levels are considered as so shallow that thermal casting of electrons into conduction band are possible.

2) Then we shell substitute the found inner field into the wave equation for high-frequency laser pump field and calculate the changes in its structure allowing for linear EO-effect. Our calculations correspond to the following geometry: the pump wave vector is directed along the crystal optical axis $\mathrm{Z}$, the pump polarization vector $\vec{e}_{p}$ lies in the XY-plane, the coordinate origin coincides with the pump beam center on the front plane of the crystal. The crystal, which has symmetry group $\mathrm{C}_{3 \mathrm{~V}}$, is considered as infinite in the transverse directions $\mathrm{X}, \mathrm{Y}$, since the pump beam diameter used in the wave equation is much smaller than the transverse crystal sizes and has definite thickness $l$ in Z-direction.

\section{The dynamics of inner field in the constant pump wave}

\subsection{The general system of equations}

In accordance with papers [13] the system of equations for the inner field in the quasi-homogeneous region of the PRC has the form:

$$
\begin{aligned}
& \frac{\partial n_{d}^{+}}{\partial t}=-\gamma m n_{d}^{+}+\left(s I_{0}+s_{0}\right)\left(n_{d}^{0}-n_{d}^{+}\right), \\
& \frac{\partial n}{\partial t}=\frac{\partial n_{d}^{+}}{\partial t}+\frac{d i v \vec{j}_{n}}{e}, \\
& S Q O, 4(4), 2001
\end{aligned}
$$

$$
\begin{aligned}
& \operatorname{div}\left(\varepsilon \vec{E}_{f}\right)=4 \pi e\left(-n_{a}-n+n_{d}^{+}\right) \\
& \vec{j}_{n}=e \mu n \vec{E}_{f}+e \operatorname{Dgrad}(n)+\left(n_{d}^{0}-n_{d}^{+}-\frac{2 \gamma n}{s I_{0}+s_{0}} n_{d}^{+}\right) s I_{0} \vec{G}
\end{aligned}
$$

Here the following designations are introduced: $n$ is the concentration of free electrons, $n_{q}$ is the concentration of acceptors, $n_{d}^{0}$ is the concentration of donors, $n_{d}^{+}$is the concentration of ionized donors (traps), $g$ is the capture coefficient of electron by trap, $s$ is the photoionization cross-section, $I_{0}$ is the pump intensity, $s_{0}$ is the thermalization coefficient, $e$ is the absolute value of the electron charge, $j_{n} \mathrm{~s}$ the density of electron current, $\varepsilon$ is static dielectric permittivity, $\mu$ is the electron mobility, $D$ is the diffusion coefficient, $G_{k}=\beta_{k l m} e_{l} e_{m}^{*}$ is the Glass vector (the double convolution of the photovoltaic tensor with the pump wave polarization $\left.e_{p}\right)$. As distinct from $[12,13]$, in (1d) we have taken into account that the concentration of photoelectrons in the conductive band, which determines the density of photovoltaic current, is less than that of electrons knocked out from not-ionized donors by light quantum. This happens owing to the capture of photoelectron by trap earlier than it reaches the conductive band. The probability of this process is $W_{t r}=$ $=2 \mathrm{~m} /\left(s I_{0}+s_{0}\right)$.

The system (1) must be supplemented by initial and boundary conditions. The latter conditions represent the field state at the sample boundaries $z=0$ and $z=l$. Let us discuss the cases of free and short-circuited samples. In the literature [14-15] one can find the detailed description of the spontaneous polarization $\left(P_{s}\right)$ screening in the uniaxial ferroelectrics without illumination. The main results of these works can be formulated as the following statements. The screening is internal for the stationary state of single-domain ferroelectric with not rather broad band gap and therefore does not noticeably influence on the distribution of the inner field and spontaneous induction in the sample. Thus in the case the short-circuited sample can be treated as the sample with free surface, and one can apply to it the solutions for the fields obtained in [15] for the free one. These solutions correspond to the small purely longitudinal constant inner field in the bulk of the crystal, i.e. at $\ell_{c}<z<\ell-\ell_{c}$ ( $l$ is the crystal thickness, $l_{c}$ is the correlation length), and sharply dependent over $z$ coordinate strong longitudinal field in the surface layer (SL), i.e. at $0<z<\ell_{c}, \quad \ell-\ell_{c}<z<\ell$. Here we would like to emphasize that the influence of the bulk photovoltaic effect negligibly small in SL because of strong symmetry disturbance near the surface and thus in this region.

The system (1) has been solved when used the perturbation theory at $\ell_{c}<<\ell$ and the aforementioned statements about the constant inner field.

1) The only stationary (s) spatial uniform solution of (1) can be chosen as zero approximation: $\vec{E}_{s}=\operatorname{const}\left(I_{0}\right) \times$ $\times \vec{G}, \quad n_{d s}^{+}=$const, $\quad n_{s}=$ const, which at $\ell_{c}<z<\ell-\ell_{c}$ is valid either for short-circuited or for free sample. It 


\section{A. N. Morozovska et al.: Dynamics of photoinduced instability in ferroelectric...}

smoothly sews together with solution [14-15] in the SL due to $\vec{G} \equiv 0$.

2) Small dynamic corrections to this stationary solution of (1) can be obtained as the first approximation: $\vec{E}_{f}=$ $=\vec{E}_{s}+\vec{E}_{f}(x, y, z, t), \quad n_{d}^{+}=n_{d s}^{+}+\tilde{n}_{d}^{+}, \quad n=n_{s}+\tilde{n}$. In order to use the aforementioned stationary solution [15] as good zero approximation, in accordance with the stability theory the transverse dynamic components of the inner field must satisfy the trivial boundary conditions in SL: $\widetilde{E}_{f, y}\left(x, y, 0<z<\ell_{c}, t\right)=\widetilde{E}_{f, y}\left(x, y, \ell-\ell_{c}<z<\ell, t\right)=0$. Only these conditions provide the longitudinal form of the field in SL and continuity of its tangential components at the crystal boundaries.

\subsection{The stationary solution of system}

Neglecting the existence of circular currents in PRC, the stationary case $(1 \mathrm{~b})$ is reduced to the condition of the neglecting of magnetic field in PRC $[9,12]: j_{n}=0$. When taking into account that all values are time independent, one nonlinear equation in particular derivatives for the concentration of free carriers $n_{S}$ can be obtained instead of (1):

$$
\begin{aligned}
& \frac{D}{\mu} \Delta\left(\ln n_{s}\right)-\frac{s I_{0} \vec{G}}{e \mu} \nabla\left(\frac{n_{d}^{0}}{n_{s}+\left(s I_{0}+s_{0}\right) / \gamma}\right)+ \\
& +\frac{4 \pi e}{\varepsilon}\left(\frac{s I_{0}+s_{0}}{m_{s}+s I_{0}+s_{0}} n_{d}^{0}-n_{s}-n_{a}\right)=0
\end{aligned},
$$

and relations:

$$
\begin{aligned}
& n_{d s}^{+}=\frac{s I_{0}+s_{0}}{m m_{s}+s I_{0}+s_{0}} n_{d}^{0}, \\
& \vec{E}_{s}=-\frac{D}{\mu} \nabla\left(\ln n_{s}\right)+\frac{s I_{0} \vec{G}}{e \mu}\left(\frac{n_{d}^{0}}{n_{s}+\left(s I_{0}+s_{0}\right) / \gamma}\right) .
\end{aligned}
$$

The boundary conditions for $n_{s}$ can be obtained after the substitution of the latter equality into the ones for the field. form:

The only spatial uniform solution of $(2,3)$ has the

$$
\begin{aligned}
& n_{s}=-\frac{n_{a}}{2}-\frac{s I_{0}+s_{0}}{2 \gamma}+ \\
& +\frac{1}{2} \sqrt{\left(\begin{array}{c}
s I_{0}+s_{0} \\
2 \gamma
\end{array}\right)^{2}+\frac{s I_{0}+s_{0}}{\gamma}\left(4 n_{d}^{0}-2 n_{a}\right)+n_{a}^{2}}, \\
& \vec{E}_{s}=-\frac{s I_{0} \vec{G}}{e \mu} \frac{m_{d s}^{+}}{\left(s I_{0}+s_{0}\right)}, \quad n_{d s}^{+}=n_{s}+n_{a} .
\end{aligned}
$$

Solution (4) exists if only $n_{d}^{0}>n_{a}$, at arbitrary physically correct values of other parameters.

Taking into consideration the discussion from item 2.1, we will use (4) as zero approximation for inner field dynamics in the bulk of the crystal.

\subsection{Linearized system for dynamic components}

Using (1a, 1c, 1d), after elementary transformations, (1b) can be rewritten as:

$$
\begin{aligned}
& \operatorname{div}\left(\frac{\varepsilon}{4 \pi} \frac{\partial}{\partial t} \vec{E}_{f}+e \mu n \vec{E}_{f}+e \operatorname{Dgrad}(n)-\right. \\
& \left.\left(n_{d}^{0}-n_{d}^{+}-\frac{2}{s I_{0}+s_{0}} \frac{\partial n_{d}^{+}}{\partial t}\right) s I_{0} \vec{G}\right)=0 .
\end{aligned}
$$

After neglecting the existence of magnetic field in PRC $[9,12]$, one can obtain that:

$$
\begin{aligned}
& \frac{\varepsilon}{4 \pi} \frac{\partial}{\partial t} \vec{E}_{f}+e \mu n \vec{E}_{f}+e \operatorname{Dgrad}(n)- \\
& -\left(n_{d}^{0}-n_{d}^{+}-\frac{2}{s I_{0}+s_{0}} \frac{\partial n_{d}^{+}}{\partial t}\right) s I_{0} \vec{G}=0 .
\end{aligned}
$$

We will find the solution of (1) in the form:

$$
\begin{array}{lll}
\vec{E}_{f}=\vec{E}_{s}+\tilde{\vec{E}}_{f}(x, y, z, t), & n_{d}^{+}=n_{d s}^{+}+\tilde{n}_{d}^{+}, & n=n_{s}+\tilde{n}, \\
\left|\tilde{\vec{E}}_{f}(x, y, z, t)\right| \ll<\left|\vec{E}_{s}\right|, & \left|\tilde{n}_{d}^{+}\right| \ll<n_{d s}^{+}, & |\tilde{n}| \ll<n_{s},
\end{array}
$$

with the boundary conditions for the inner field:

$$
\begin{aligned}
& \tilde{E}_{f_{x, y}}\left(x, y, 0<z<\ell_{c}, t\right)=\tilde{E}_{f_{x, y}}\left(x, y, \ell-\ell_{c}<z<\ell, t\right)=0, \\
& \tilde{E}_{f_{z}}(x, y, z=\tilde{\ell}, t)=v(x, y, t), \quad \ell_{c}<\tilde{\ell}<\ell-\ell_{c},
\end{aligned}
$$

here $v(x, y, t) \sim I_{0}$ is small photoinduced fluctuations with rather broad spatial-temporal spectrum.

Representation (7) allows us to linearize the system for small dynamic components. In the first approximation the properties of the solutions of (1) should be agreed with the stability theory: at the stability threshold the dynamic components have to be not increasing functions of time $t$ and transverse coordinates $x, y$ and analytical functions of $z$, i.e. the Fourier image at variables $t, x, y$ and the Laplace image at variable $z$ must exist. Then:

$$
\begin{aligned}
& \tilde{\vec{E}}_{f}(x, y, z, t)=\int d \vec{q} d \Omega \cdot \vec{U}(\vec{q}, \Omega) \exp (i \vec{q} \vec{r}+i \Omega t), \\
& \tilde{n}(x, y, z, t)=\int d \vec{q} d \Omega \hat{n}(\vec{q}, \Omega) \exp (i \vec{q} \vec{r}+i \Omega t), \\
& \tilde{n}_{d}^{+}(x, y, z, t)=\int d \vec{q} d \Omega \cdot \hat{n}_{d}^{+}(\vec{q}, \Omega) \exp (i \vec{q} \vec{r}+i \Omega t), \\
& \text { where } \vec{q} \vec{r}=q_{z} z+q_{x} x+q_{y} y, \quad q_{z}=q_{z}^{\prime}+i q_{z}^{\prime \prime}, \\
& \left(q_{z}^{\prime}, q_{z}^{\prime \prime}, q_{x}, q_{y}, \Omega\right) \in(-\infty, \infty) . \\
& i \Omega \hat{n}_{d}^{+}=-\hat{n} n_{d s}^{+}-\left(s I_{0}+s_{0}+m_{s}\right) \hat{n}_{d}^{+}, \\
& i(\vec{q} \vec{U})=\frac{4 \pi e}{\varepsilon}\left(\hat{n}_{d}^{+}-\widehat{n}\right), \\
& \frac{\varepsilon}{4 \pi} i \Omega \vec{U}+e \mu\left(n_{s} \vec{U}+\widehat{n} \vec{E}_{s}\right)+ \\
& +i e D \vec{q} \hat{n}+\left(1+\frac{2 i \Omega}{s I_{0}+s_{0}}\right) \widehat{n}_{d}^{+} s I_{0} \vec{G}=0 .
\end{aligned}
$$




\section{A. N. Morozovska et al.: Dynamics of photoinduced instability in ferroelectric...}

$$
\begin{aligned}
& \hat{n}_{d}^{+}=\frac{i \varepsilon n_{d s}^{+}(\vec{q} \vec{U})}{4 \pi e\left(i \Omega+s I_{0}+s_{0}+\gamma\left(n_{s}+n_{d s}^{+}\right)\right)}, \\
& \hat{n}_{d}^{+}=-\frac{i \varepsilon\left(i \Omega+s I_{0}+s_{0}+m_{s}\right)(\vec{q} \vec{U})}{4 \pi e\left(i \Omega+s I_{0}+s_{0}+\gamma\left(n_{s}+n_{d s}^{+}\right)\right)},
\end{aligned}
$$

$$
\begin{aligned}
& \vec{U}\left(\frac{\varepsilon}{4 \pi} i \Omega+e \mu n_{s}\right)-i \varepsilon(\vec{q} \vec{U}) \times \\
& \times \frac{\left(i e D\left(i \Omega+s I_{0}+s_{0}+m_{s}\right) \vec{q}-\frac{\hat{n}_{d}^{+} s I_{0}\left(i \Omega-m_{s}\right) \vec{G}}{s I_{0}+s_{0}}\right)}{4 \pi e\left(i \Omega+s I_{0}+s_{0}+\gamma\left(n_{s}+n_{d s}^{+}\right)\right)}=0 .
\end{aligned}
$$

Before solving the latter vector equation let us rewrite it in appropriate variables. Then Eq. (11b) acquires the form:

$$
\begin{aligned}
& \vec{U}\left(1+i \Omega \tau_{m}\right)+ \\
& +\frac{(\vec{q} \vec{U})\left(\ell_{d}^{2}\left(i \Omega \tau_{s}+f\right) \vec{q}-\delta\left(\Omega \tau_{s}+i(f-1)\right) \ell_{g}\right)}{i \Omega \tau_{s}+f+\tau_{s} / \tau_{d}}=0,
\end{aligned}
$$

where new variables are introduced $\tau_{m}=\varepsilon / 4 \pi e \mu n_{s}$ is the maxwellian time, $\tau_{d}=1 / \gamma n_{d s}^{+}$is the time of electron capture by trap, $\tau_{s}=1 /\left(s I_{0}+s_{0}\right)$ is the photoionization time, $\ell_{d}=\sqrt{\varepsilon D / 4 \pi e \mu n_{s}}$ is the diffusion length (if the Einstein ratio is valid $e D=\mu k_{B} T$, the diffusion length coincides with Debye screening radius [14]), $\vec{\ell}_{g}=\varepsilon n_{d s}^{+} \vec{G} / 4 \pi e^{2} \mu n_{s}$ the photovoltaic (drift) length, $f=n_{d}^{0} / n_{d s}^{+}$is the ratio of donors concentration to traps one $(f>1), \delta=s I_{0} /\left(s I_{0}+\right.$ $\left.+s_{0}\right)$.

Scalar product of (12) by $\vec{q}$ gives us the dispersion equation:

$$
1+i \Omega \tau_{m}+\frac{\ell_{d}^{2}\left(i \Omega \tau_{s}+f\right) \vec{q}^{2}-\delta\left(\Omega \tau_{s}+i(f-1)\right)\left(\vec{\ell}_{g} \vec{q}\right)}{i \Omega \tau_{s}+f+\tau_{s} / \tau_{d}}=0 .
$$

When solving (12) for different components, the components $U_{x, y}$ can be expressed through $U_{z}$ :

$$
U_{x, y}=\frac{U_{z}\left(\ell_{d}^{2}\left(i \Omega \tau_{s}+f\right) q_{x, y}-\delta \cdot\left(\Omega \tau_{s}+i(f-1)\right) \ell_{g x, y}\right)}{\ell_{d}^{2}\left(i \Omega \tau_{s}+f\right) q_{z}-\delta \cdot\left(\Omega \tau_{s}+i(f-1)\right) \ell_{g z}} .
$$

Taking into account that $q_{x, y}, U_{z}, l_{\mathrm{d}}$ cannot be equal to zero in SL, (14) satisfies the boundary conditions (8) only if $U_{x, y} \equiv 0$. The vector $q_{x, y}$ can be found from (14). Its components must be real as the components of Fourier transform wave vector (9). Thus, the expression for $\Omega_{f}$ can be found from the condition $\operatorname{Im}\left(q_{f x, y}\right)=0$. The expression for $q_{f z}$ was found from (13) after substitution $\Omega_{f}$, $q_{f x, y}$. As the result we obtained:

$$
\begin{aligned}
& \Omega_{f}= \pm \frac{\sqrt{f(f-1)}}{\tau_{s}}, \quad q_{f x, y}= \pm \delta \sqrt{1-1 / f} \frac{\vec{\ell}_{g x, y}}{\ell_{d}^{2}} \\
& \ell_{d}^{2}\left(i \Omega_{f} \tau_{s}+f\right) q_{f z}^{2}-\delta\left(\Omega_{f} \tau_{s}+i(f-1)\right) \ell_{g z} q_{f z}+ \\
& +\left(1+i \Omega_{f} \tau_{m}\right)\left(i \Omega_{f} \tau_{s}+f+\tau_{s} / \tau_{d}\right)=0 .
\end{aligned}
$$

If the following inequalities are valid: $l_{g}>>l_{d}$, and $t_{s} / t_{d} \times$ $\times\left(f+i \Omega_{f} t_{S}\right)<<1$, the expression for $q_{f z}$ is simplifies into the form:

$$
q_{f z}=\frac{1}{\delta \ell_{g z}}\left( \pm \sqrt{\frac{f}{f-1}}-i f \tau_{m} / \tau_{s}\right)=q_{f z}^{\prime}-i q_{f z}^{\prime \prime} .
$$

The signs in $(15,16)$ must be upper or lower.

Let's study the dependence of frequency $\Omega_{f}$ and wave vector $q_{f}$. from $(15 \mathrm{a}, 16)$ over pump intensity $I_{0}$. For this purpose we introduced the following dimensionless variables and functions:

$$
\begin{aligned}
& \Omega_{s}(\xi)=\xi \sqrt{f^{2}(\xi)-f(\xi)}, \\
& f(\xi)=\frac{\sqrt{\xi^{2}+\xi(4-2 h)+h^{2}}+\xi-h}{2 \xi}, \\
& q_{s z}^{\prime}(\xi)=\frac{\xi(1-h f(\xi))}{\xi-\chi} \sqrt{\frac{f(\xi)}{f(\xi)-1}}, \quad q_{s z}^{\prime \prime}(\xi)=f^{2}(\xi) \frac{\xi^{2}}{\xi-\chi}, \\
& q_{s \perp}(\xi)=\frac{\xi-\chi}{\xi} \sqrt{\frac{f(\xi)-1}{f^{3}(\xi)}} \\
& \Omega_{s}(\xi)=\Omega_{f} / m_{d}^{0}, \quad q_{s z}^{\prime}(\xi)=q_{f z}^{\prime} \varepsilon \gamma \quad G_{z} /\left(4 \pi e^{2} \mu\right), \\
& q_{s z s}^{\prime \prime}(\xi)=q_{f z}^{\prime \prime} G_{z} / e, \quad q_{s \perp}(\xi)=e D q_{f x, y} /\left(m_{d}^{0} G_{x, y}\right), \\
& \xi=\left(s I_{0}+s_{0}\right) / m_{d}^{0}, \quad h=n_{a} / n_{d}^{0}, \quad \chi=s_{0} / m_{d}^{0} .
\end{aligned}
$$

Formula (17), as well as the solution (2.4), is valid if $h<1$ at arbitrary values of other parameters. The expansions for $\Omega_{s}$ and $q_{s}$ at small (but from (17) one can get than $\xi \geq \chi)$ and big $\xi$, i.e. at small and high pump wave intensities, can be easily obtained from (17):

$$
\begin{array}{ll}
\Omega_{s}(\xi \rightarrow 0) \approx \xi \frac{\sqrt{1-h}}{h}, & \Omega_{s}(\xi \rightarrow \infty) \approx \sqrt{\xi(1-h)}, \\
q_{\perp s}(\xi \rightarrow 0) \approx \frac{\xi-\chi}{\xi} h \sqrt{1-h}, & q_{s \perp}(\xi \rightarrow \infty) \approx \sqrt{\frac{(1-h)}{\xi}} .
\end{array}
$$

Analogous expansions can be obtained for the imaginary and the real parts of gain factor $q_{s z}$ :

$$
\begin{aligned}
& q_{s z}^{\prime \prime}(\xi \rightarrow 0) \approx \frac{\xi^{2}}{h^{2}(\xi-\chi)}, \\
& q_{s z}^{\prime \prime}(\xi \rightarrow \infty) \approx \xi+2-2 h+\chi, \\
& q_{s z}^{\prime}(\xi \rightarrow 0) \approx \frac{\xi^{2}\left((2-h)^{2} / h^{3}-1\right)}{4(\xi-\chi) \sqrt{1-h}}, \\
& q_{s z}^{\prime}(\xi \rightarrow \infty) \approx \sqrt{(1-h) \xi} .
\end{aligned}
$$




\section{A. N. Morozovska et al.: Dynamics of photoinduced instability in ferroelectric...}

In accordance with (18a), the dependence of frequency $\Omega_{s}$ over intensity and high intensities obeys the energy conservation law in the system «classical oscillator with the frequency $\Omega_{s}$ (as the energy transformer) + pump field with intensity $I_{0}$ (as source of energy )». At rather small intensities the frequency is proportional to the «renormalized» intensity $\xi$. The numerical calculation shows us that at intermediate $\xi$ values the aforementioned dependence can also be rather well approximated by linear function but with another coefficients than at small $\xi$.

The dependences of dimensionless frequency $\Omega_{s}$ and transverse wave vector $q_{s \perp}$ over renormalized intensity $\xi$ are depicted in Fig. 1.

Thus, based on $(7,9,15-16)$, we can rewrite the solution of the inner field in the form:

$$
\begin{aligned}
& \vec{E}_{f}(x, y, z, t)=\vec{E}_{s}+\vec{e}_{z}\left(U \operatorname { e x p } \left(i \Omega_{f} t+i \vec{q}_{f \perp} \vec{r}_{\perp}+\right.\right. \\
& \left.\left.+\left(i q_{f z}^{\prime}+q_{f z}^{\prime \prime}\right)(z-\tilde{\ell})\right)+c . c\right), \\
& \vec{E}_{s}=\vec{E}_{0} \frac{\delta}{f}, \quad \vec{E}_{0}=\frac{m_{d}^{0}}{e \mu} \vec{G}, \quad U<E_{s}, \quad \ell_{c}<z<\ell-\ell_{c} .
\end{aligned}
$$

In (19) the sign «+» must be chosen in expressions $(15,16)$ for the frequency, and the wave vector. $U=R_{F}\left(q_{f x, y}, \Omega_{f}\right)$ is the Fourier image of fluctuation spectrum (8) at $q_{f x, y}=$ $=q_{x, y}, \Omega=\Omega_{f}$.

Since all material parameters of the crystal have been determined constants. Substantially they vary because of the polarization vector rotation due to the birefringence (which leads to the rotation of the vector $G_{x, y}$, considered in item 3.3) and such growth peculiarities as non-homogeneous in transverse directions (sometimes similar to spiral) impurity distribution (i.e. the fluctuations of $n_{a}$, $n_{d}$ ), which lead to the floating $f$ the crystallographic axes $X$ due to the local symmetry breaking near defects (i.e. the fluctuations of $G_{x, y}$ ) [16]. That is why the whole set of frequencies and wave vectors are present in the real system. Note that our theory is not valid for $I_{0}=0$, because, as it follows from $(17,18), E_{s}\left(I_{0}=0\right)=0$, i.e. the nontrivial zero approximation does not exist.

As the conclusion of this item, we can predict that at not very high $I_{0} G$ (but $I_{0} \neq 0$ ) and $l_{g x, y} \sim G_{22} \sim b_{22} \neq 0$ (because $\left.q_{f x, y} \sim 1_{g x, y}\right)$, the boundary cycle exists in the nonlinear system (1) in accordance with the Wiener-Hopf theorem. Its spectrum has maximum at $|\Omega|=\Omega_{f}, q_{x, y}= \pm q_{f x, y}$, i.e. the completion degree of traps varies periodically both in time and in space. This means that the stable recharging waves of traps can propagate in the transverse crystal direction. The final results must be averaged over all fluctuating values. For our theory this result is the laser field changed by interaction with the PRC in the far zone from the crystal. Therefore it is necessary to average only the final diffraction pattern (see the next item).

\section{Dynamic photoinduced light scattering by PRC}

\subsection{Dynamics of laser fields in PRC in the approximation of prescribed inner field}

The system of equations, which describes the interaction of laser pump wave with the inner field found in item 2 (i.e. with the field generated by this wave itself in the
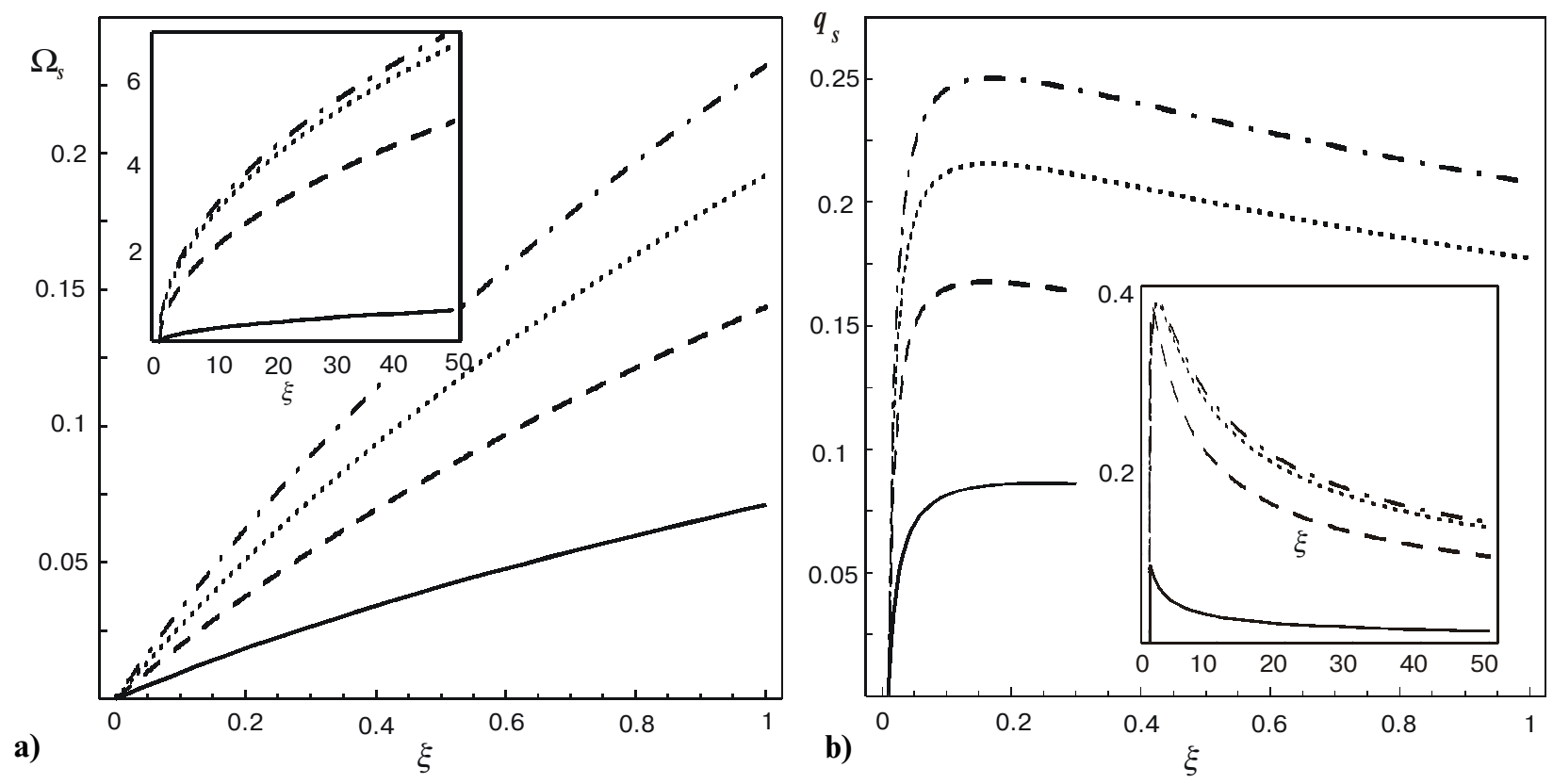

Fig. 1. The dependence of $\Omega_{\mathrm{s}}(\mathrm{a}), \mathrm{q}_{\mathrm{s} \perp}$ (b) over $\xi$ at $\chi=0.01$ at different $h$ values: $h=0.90$ (dash-dotted curves); 0.96 (dotted curves); 0.93 (dashed curves); 0.99 (solid curves). In the insets $h=0.01$ (dash-dotted curves); 0.1 (dotted curves); 0.5 (dashed curves); 0.99 (solid curves). 


\section{A. N. Morozovska et al.: Dynamics of photoinduced instability in ferroelectric...}

nonlinear medium) in the PRC, will be considered within the framework of the general theory of mathematical diffraction [17] when taking into account the linear EOeffect.

Keeping in mind the geometry of the problem (see Ph.1), the time-independent complex amplitude of the pseudopotential $\varphi$ of the laser field $E^{\omega}=(-\operatorname{grad}(\varphi) \times$ $\times \exp (-i \omega t)+$ c.c. $)$ can be represented as the sum of the following amplitudes:

1) $A_{0}(x, y, z) \exp \left(i k_{0} z\right)$ is the amplitude of the pump wave pseudopotential before the incidence on the front plane of the crystal, i.e. at $z \leq 0$;

2) $A_{1}(x, y, z, t) \exp \left(i k_{\varepsilon} z\right)$ is the amplitude of the deflected wave pseudopotential propagating in the crystal at $0 \leq z \leq l$, i.e. the amplitude of the direct wave;

3) $B_{1}(x, y, z, t) \exp \left(i k_{0} z\right)$ is the amplitude of the pump wave pseudopotential after penetration through the crystal, i.e. $z \geq l$;

4) $A_{2}(x, y, z, t) \exp \left(i k_{\varepsilon}\left(2\left(l-l_{c}\right)-z\right)\right)$ is the amplitude of the pseudopotential in the crystal of the wave reflected from the SL on its back plane, i.e. the amplitude of the inverse wave at $l \geq z \geq 0$;

5) $\left(B_{2}(x, y, z, t)+B_{0}(x, y, z),\right) \exp \left(-i k_{0} z\right)$ are the amplitudes of the pseudopotential of the inverse wave after transmission through the crystal and the part of the pump wave reflected from its front plane correspondingly at $z \leq 0$.

Hereinafter we use the definitions: $k_{0}=\sqrt{\varepsilon_{0}^{\omega}} \omega / c$ is the high-frequency part of the laser waves with wave vector outside the sample, $k_{\varepsilon}=k_{0} \sqrt{\varepsilon}$ is the same value inside the PRC. Allowing for EO-effect the crystal refractive index $\varepsilon=\varepsilon^{\omega}+\Delta \varepsilon\left(E_{f}\right)+\delta \varepsilon$, where $\Delta \varepsilon_{i j}\left(E_{f}\right)=\chi_{i j k} E_{f k}, \delta \varepsilon$ are small fluctuations.

The equations for the smoothly changing parts of the direct and inverse wave amplitudes in the crystal $\left(A_{1,2}\right)$ one can obtain from the wave equation for pseudopotential in paraxial approximation [18]. They have the form:

$$
\begin{aligned}
& \frac{\partial A_{1}}{\partial z}-i p \Delta_{\perp} A_{1}=i w A_{1}, \\
& -\frac{\partial A_{2}}{\partial z}-i p \Delta_{\perp} A_{2}=i w A_{2},
\end{aligned}
$$

where $p=1 / 2 k_{\varepsilon}$ and $w=k_{\varepsilon}\left(\chi_{13} E_{f z}+\chi_{22}\left(E_{f y}-E_{f x}\right)\right) / 2\left(\chi_{13}\right.$ is the component of EO-tensor). The pump amplitude $A_{0}$ satisfies (1a) at $w=0$. Taking into consideration that in our theory $l>l_{c}$, we suppose that the passing through the extremely thin SL does not make any significant contribution in the all aforementioned amplitudes. Thus, in further consideration, we can neglect the thickness of this layer, however keeping in mind that the generation of the inverse wave is «internal», i.e. is caused by the photorefractive jump of $\Delta \varepsilon$ at the boundary of screening region and double electric layer. This generation is not «external» or taking place on the geometric boundary of the sample, where the representation (2.19) for inner field is not valid. All external reflections can be avoided after putting the sample into immersion liquid. Therefore, the existence of the aforementioned dynamic effects is independent on the refractive index of the outer medium.

The boundary conditions for $A_{1,2}$ can be obtained from the conjugation conditions [17] for $\varphi$, i.e. the continuity of $\varphi$ and its normal derivatives at crystal boundaries $z=0, l$. After rewriting $B_{0}(0)$ in terms of $A_{1,2}(0)$ and $B_{1}(l)$ in terms of $A_{1,2}(l)$ we obtain:

$$
\begin{aligned}
& A_{1}(x, y, z=0, t)=\frac{2}{n_{0}+1} A_{0}(x, y, z=0), \\
& A_{2}(x, y, z=\ell, t)=\frac{n-1}{n_{0}+1} A_{1}(x, y, z=\ell, t)+ \\
& +\frac{1}{2 i k_{0}(n+1)} \frac{\partial A_{1}(x, y, z=\ell, t)}{\partial z}
\end{aligned}
$$

where $n_{0}=\sqrt{\varepsilon^{\omega}} / \sqrt{\varepsilon_{0}^{\omega}}$ for laser frequency $w$, but $n \rightarrow 1$ because it is the relative high-frequency refractive index on the internal boundary of SL. Thus, in order to maintain a small seeding for the weak inverse wave, we can neglect small terms like $k_{0}{ }^{-1} \partial A_{0,1,2} / \partial z$ everywhere except the right-hand side of (2b). Note that condition $\left|A_{2}\right|<<\left|A_{1}\right|$, following from $(1,2)$, is necessary for the applicability of the non-depleted pump wave approximation used in item 2. Then, using Eq. (19) from item 2 and the perturbation theory, the solution of (1) will be found in the form analogous to that for $w$ one:

$$
\begin{aligned}
& w=v+\left(u \operatorname { e x p } \left(i \Omega_{f} t+i \vec{q}_{f \perp} \vec{r}_{\perp}+\left(i q_{f z}^{\prime}+q_{f z}^{\prime \prime}\right)(z-\tilde{\ell})+\right.\right. \\
& \left.\left.++i \varphi_{f}\right)+c . c\right), \quad|v|>>u, \\
& A_{1,2}(\vec{r}, t)=a_{1,2}(\vec{r})+b_{1,2}^{+}(\vec{r}) \exp \left(i \Omega_{f} t+i \vec{q}_{f \perp} \vec{r}_{\perp}+\right. \\
& \left.+\left(i q_{f z}^{\prime}+q_{f z}^{\prime \prime}\right)(z-\tilde{\ell})+i \varphi_{f}\right)+b_{1,2}^{-}(\vec{r}) \exp \left(-i \Omega_{f} t-\right. \\
& \left.-i \vec{q}_{f \perp} \vec{r}_{\perp}+\left(-i q_{f z}^{\prime}+q_{f z_{f}}^{\prime \prime}\right)(z-\tilde{\ell})-i \varphi_{f}\right), \\
& \left|\Delta_{\perp} a_{1,2}(\vec{r})\right|<<\left|q_{f \perp}^{2} a_{1,2}(\vec{r})\right|,\left|\Delta_{\perp} b_{1,2}^{ \pm}(\vec{r})\right|<<\left|q_{f \perp}^{2} b_{1,2}^{ \pm}(\vec{r})\right|,
\end{aligned}
$$

i.e. $a_{1,2}, b_{1,2}$ are supposed to be rather smooth functions of transverse coordinates. $\varphi_{f}$ is the phase related with the choice $u>0$. After neglecting of multiple harmonics two systems of equations for $a_{1,2}, b_{1,2}$ can be obtained from $(1,3)$ :

$$
\begin{aligned}
& \pm \frac{\partial a_{1,2}}{\partial z}-i p \Delta_{\perp} a_{1,2}=i\left(v a_{1,2}+u \exp \left(2 q_{f z}^{\prime \prime}(z-\tilde{\ell})\right)\left(b_{1,2}^{+}+b_{1,2}^{-}\right)\right), \\
& \pm \frac{\partial b_{1,2}^{+}}{\partial z} \pm\left(i q_{f z}^{\prime}+q_{f z}^{\prime \prime}\right) b_{1,2}^{+}+i p q_{f \perp}^{2} b_{1,2}^{+}=i\left(v b_{1,2}^{+}+u a_{1,2}\right), \\
& \pm \frac{\partial b_{1,2}^{-}}{\partial z} \pm\left(-i q_{f z}^{\prime}+q_{f z}^{\prime \prime}\right) b_{1,2}^{-}+i p q_{f \perp}^{2} b_{1,2}^{-}=i\left(v b_{1,2}^{-}+u a_{1,2}\right) .
\end{aligned}
$$

The sign «+» in (4) corresponds to the system for the direct wave with subscript 1 , and the sign «-» corresponds to the system for the inverse wave with subscript 2 . The 


\section{A. N. Morozovska et al.: Dynamics of photoinduced instability in ferroelectric...}

boundary conditions derived from $(2,3)$ have the following appearance in our approximations:

$$
\begin{aligned}
& a_{1}(x, y, z=0)=\frac{2}{n_{0}+1} A_{0}(x, y, z=0), \quad b_{1}^{ \pm}(x, y, z=0)=0, \\
& a_{2}(x, y, z=\ell)=\frac{n-1}{n+1} a_{1}(x, y, z=\ell) \rightarrow 0, \\
& b_{2}^{ \pm}(x, y, z=\ell)=\frac{\Delta n^{ \pm}+n-1}{n+1} b_{1}^{ \pm}(x, y, z=\ell), \\
& \Delta n^{ \pm}=\frac{ \pm q_{f z}^{\prime}-i q_{f z}^{\prime \prime}}{k_{0}} .
\end{aligned}
$$

The components $b_{1,2}$ can be easily expressed in terms of $a_{1,2}$ by means of (4) and (5). Then we are to solve two linear integral-differential equations for $a_{1,2}$ with the appropriate boundary conditions. Unfortunately, we failed in finding their precise solution. Again we solved them in the perturbation theory method with respect to the small parameter $u$. In the zero approximation the «side» components $b_{1,2}$ are identically equal to zero, and only the non-depleted pump wave exists. In the first approximation «central» components $a_{1,2}$ do not deplete, and «side» components $b_{1,2}$, reinforce under passing through the crystal in the photoinduced bleaching regime (see below the comments to (6)). Thus, the constant pump approximation for the inner field is self-consistent. In the second and all higher approximations, «central» components deplete, and «side» components reinforce, i.e. the second and higher approximations do not contradict with the conservation laws. Indeed, the energy of the pump wave is expended on the creation and amplification of the inner field and side components. It is impossible to convolute the above-mentioned perturbation series exactly, one can prove its convergence and use «Feynman trick» to obtain the approximate expression with the accuracy up to $u^{4}$ terms.

This final result for the amplitudes $A_{1,2}$ of laser fields in the crystal can be represented in the form: where $f_{1,2}(z)$ are rather cumbersome elementary functions arisen under the approximate convolution of the series over $u, z^{ \pm}(z)$ are the «mean points» arisen due to using the Lagrange theorem in the calculation of integrals over variable $z$ at products of the functions of $z$ and $A_{0}(x, y, z)$.

As it follows from (6), that $\left|R_{2}^{ \pm}(0)\right| \gg>\left|R_{2}^{ \pm}(\ell)\right|$ and $\left|R_{1}^{ \pm}(\ell)\right| \gg>\left|R_{1}^{ \pm}(0)\right|$ if only $q_{f z}^{\prime \prime} \ell<<0$. Therefore, «side» components reinforce significantly under passing through the crystal in the photoinduced bleaching regime. The largest amplification of the inverse wave corresponds to its generation near the back plane, i.e. at $\tilde{\ell} \approx \ell-\ell_{c} \approx \ell$. In that case the following approximation equalities are valid: $R_{2}^{ \pm}(0) \approx R_{2}^{ \pm}(\ell) \exp \left(-2 q_{f z}^{\prime \prime} \ell\right), \quad R_{1}^{ \pm}(\ell) \approx R_{1}^{ \pm}(0) \exp \left(-q_{f z}^{\prime \prime} \ell\right)$. Based on Eq. (19) from item 2, the fluctuations near the back plane will exactly have the maximum amplification coefficient in the photoinduced bleaching regime. And so the considered dynamic effects can be registered, if the direction of the pump wave vector coincides with the crystal polar axis, i.e. in the photoinduced bleaching regime.

The obtained results (6) are valid for any rather smooth in the central region of the sample pump wave profile $A_{0}(x, y, z)$. This smooth function was approximated by constant in item 2 and supposed that intensity is negligibly small and therefore didn't effect on the inner field formation in the «fringe» region, where it is impossible to neglect its coordinate dependence. For example, the gaussian profile of the pump wave is quite suitable for all aforementioned demands. Namely, in this case we can affirm, that the recording of dynamic holographic grating by pump wave (i.e. the creation of profiling inner field) and the amplification of the "side» components under their diffraction on the grating do not change the spatial structure of the pump wave in the first approximation, but only slightly decrease its intensity.

$$
\begin{aligned}
& A_{1}(x, y, z, t)=S_{1}(z)\left(A_{0}(x, y, z)+\sum_{ \pm} R_{1}^{ \pm}(z) A_{0}\left(x, y, \zeta^{ \pm}(z)\right) \exp \left( \pm i\left(\Omega_{f} t+q_{f x} x+q_{f y} y\right)\right)\right. \\
& A_{2}(x, y, z, t)=S_{2}(z)\left(\begin{array}{l}
R_{0} A_{0}(x, y, 2 \ell-z)+ \\
\sum_{ \pm} R_{2}^{ \pm}(z) A_{0}\left(x, y, \zeta^{ \pm}(2 \ell-z)\right) \exp \left( \pm i\left(\Omega_{f} t+q_{f x} x+q_{f y} y\right)\right)
\end{array}\right) \\
& S_{1}(z)=\frac{2 \exp \left(i v z-u^{2} \phi_{1}(z)\right)}{n_{0}+1}, \quad S_{2}(z)=\frac{2 \exp \left(i v(2 \ell-z)-u^{2} \phi_{2}(z)\right)}{(n+1)\left(n_{0}+1\right)}, \\
& R_{0}=n-1, \quad R_{1}^{ \pm}(z)=i u \exp \left(\left(q_{f z}^{\prime \prime} \pm i q_{f z}^{\prime}\right)(z-\tilde{\ell}) \pm i \varphi_{f}\right) \psi\left(-q_{f z}^{\prime \prime} \mp i q_{f z}^{\prime}-i p q_{f \perp}^{2}, z\right), \\
& R_{2}^{ \pm}(z) \cong i u\left(\Delta n^{ \pm}+n-1\right) \exp \left(\left(q_{f z}^{\prime \prime} \pm i q_{f z}^{\prime}\right)(\ell-\tilde{\ell})+i p q_{f \perp}^{2}(z-\ell) \pm i \varphi_{f}\right) \psi\left(-q_{f z}^{\prime \prime} \mp i k_{f}^{\prime}-i p q_{f \perp}^{2}, 2 \ell-z\right), \\
& \psi(d, z)=\frac{\exp (d z)-1}{d}, \quad n \rightarrow 1 \Rightarrow n^{ \pm}>>n-1, \quad \zeta^{ \pm}(z) \in(0, z), \quad \operatorname{Re}\left(\phi_{1}(z)\right)>0, \quad \operatorname{Re}\left(\phi_{2}(z)\right)<0
\end{aligned}
$$




\section{A. N. Morozovska et al.: Dynamics of photoinduced instability in ferroelectric...}

As it follows from (6) at $n=1$ the direct wave consists of the bright central beam and the weak side components, and the inverse wave consists only of the side components, because for the validity of our theory we have to require $\left|A_{1} / A_{0}-1\right|<<1$ and $\left|A_{2} / A_{0}\right|<<1$.

\subsection{Diffraction patterns on the screen}

Let us consider the aforementioned laser fields transformed by the PRC far from it, i.e. at $z=-L$ (inverse scattering) and at $z=L$ (direct scattering), where $l<<\mathrm{L}$. Hereinafter we suppose that $n=1$.

In accordance with the theory of diffraction, in order to obtain the far field on the screen $S$ with the coordinates $X_{s}, Y_{s}$ from (6), it is necessary to perform the Kirchhof transform [19] for the amplitudes of direct $E_{1}(x, y, z=l, t)$ and inverse $E_{2}(x, y, z=0, t)$ fields correspondingly over variables $x, y$. In the case of small-angle diffraction (i.e. at $\left.\theta=\sqrt{X_{s}^{2}+Y_{s}^{2}} / L<<1\right)$ and rather big $L>k_{0} \rho_{0}^{2}$ the Kirchhof transform reduces to the Fourier transform of the fields over variables $x, y$, in which $q_{x} \rightarrow k_{0} q_{x}$ and $q_{y} \rightarrow k_{0} q_{y}$, where $q_{x}=X_{s} / L, q_{y}=Y_{s} / L$ are the angle variables. Also the image must be multiplied by $k_{0} / L$. After this transformations one can derive from (6) the amplitudes of the fields on the screen at $z= \pm L$ :

$$
\begin{aligned}
& E_{1}^{L}(\vec{\theta}, t)=S_{1}(\ell)\left(F_{0}\left(k_{0} \vec{\theta}, \ell\right)+\right. \\
& \left.+\sum_{ \pm} R_{1}^{ \pm}(\ell) F_{0}\left(k_{0} \vec{\theta} \mp \vec{q}_{f \perp}, \zeta^{ \pm}(\ell)\right) \exp \left( \pm i \Omega_{f} t\right)\right) \\
& E_{2}^{L}(\vec{\theta}, t)=S_{2}(0)\left(\sum_{ \pm} R_{2}^{ \pm}(0) F_{0}\left(k_{0} \vec{\theta} \pm \vec{q}_{f \perp}, \zeta^{ \pm}(2 \ell)\right)-\right. \\
& \left.-\exp \left( \pm i \Omega_{f} t\right)\right) \\
& n=1, \quad \vec{\theta}=\left(\theta_{x}, \theta_{y}\right), \quad F_{0}\left(k_{0} \vec{\theta}, z\right) \div A_{0}\left(\vec{r}_{\perp}, z\right) .
\end{aligned}
$$

In what follows the arguments in functions $S_{1,2} R_{1,2}$ will be omitted for the sake of simplicity.

Let us choose the pump profile $A_{0}$ in the form of focused principal laser mode $\mathrm{TEM}_{00}$. Its Fourier image $F_{0}$ is the following:

$$
\begin{aligned}
& F_{0}(\vec{q}, z)=\pi \rho_{0}^{2} \sqrt{I_{0}\left(1+i n_{f}(z)\right)} \exp \left(-\frac{q^{2} \rho_{0}^{2}\left(1+i n_{f}(z)\right)}{2}\right), \\
& n_{f}(z)=\frac{z-z_{0}}{k_{0} \rho_{0}^{2}},
\end{aligned}
$$

here the beam diameter $\rho_{0}$ and the focus position $z_{0}$ are determined by the parameters of the focusing lens, $n_{f}(z)$ is the Frenel number [18].

After substituting (8) into (7) and introducing dimensionless variables one can obtain the following expres- sions for the scattering indicatrix (normalized intensity) of the direct (subscript 1) and the inverse (subscript 2) waves:

$$
j_{1}(\vec{\theta}, \tau)=\mid \exp \left(-\eta^{2}\left(1+i n_{f}\right) \theta^{2} / 2\right)+
$$

$\sum_{ \pm} R_{1}^{ \pm} \exp \left( \pm i \tau-\eta^{2}\left(1+i n_{f 1}^{ \pm}\right)\left(\vec{\theta} \mp \vec{\theta}_{f}\right)^{2} / 2\right)^{2}$

$j_{2}(\vec{\theta}, \tau)=\left|\sum_{ \pm} R_{2}^{ \pm} \exp \left( \pm i \tau-\eta^{2}\left(1+i n_{f 2}^{ \pm}\right)\left(\vec{\theta} \pm \vec{\theta}_{f}\right)^{2} / 2\right)\right|^{2}$,

$\eta=k_{0} \rho_{0}, \quad \vec{\theta}_{f}=\vec{q}_{f \perp} / k_{0}, \quad n_{f j}^{ \pm}=n_{f}\left(\zeta^{ \pm}(j \cdot \ell)\right)$,

$n_{f}=n_{f}(\ell), \quad \tau=\Omega_{f} t$.

Note that absolute values of seeding $R_{1,2}$ must be chosen in order to provide the small intensities of the side components with respect to the pump intensity. The analysis of (9) shows that the central maximum at the angle $\theta=0$ dominates in the direct scattering, and it is absent in the inverse scattering where only the «shifted» or side maxima remain at the angles $\theta_{x, y}= \pm \theta_{f x, y}$.

The formulae $(7,9)$ represent field amplitudes and scattering intensity on the screen for the ideal crystal without polarization rotation, i.e. for strictly determined value and direction of grating vector $q_{f}$. On the screen, the side maxima (7) look like two diametrically opposite spots, that appear periodically and slightly move, in other words, they are «flickering» (the effect is visible particularly well for the inverse scattering, see Fig. 2a).

The pump polarization rotation and various bulk growth defects are present in different regions of the real sample. Actually every such region has its own Glass vector, i.e. the components $q_{f \perp}$ and coefficient $R_{1,2}$, and it contributes into resulting scattering amplitude in accordance with (7). That is why for real samples (7) can be considered as the contribution from one region. In order to obtain the real field on a screen, we are to take into consideration the polarization rotation and the properties of non-homogeneities, i.e. we should sum (7) over different values $q_{f \perp}$ and $R_{1,2}$.

\subsection{The averaging of the far fields over the pump wave polarization. The models of non- homogeneities in PRC}

Let us consider the single-domain ferroelectric PRC with birefringence and fluctuations of photoactive impurity (see item 2.3, [16]). If the light propagates along the optical axis there is no natural birefringence, only its photoinduced delay can be registered [16].

For «good» samples, one can neglect fluctuations of the impurity (they do not exceed a few percents), but the rotation of the pump polarization vector $e_{p}$ always exists. Below we study the change of Glass vector components $G_{k}=\beta_{k l m} e_{l} e_{m}^{*}$ caused by this effect. 
a)

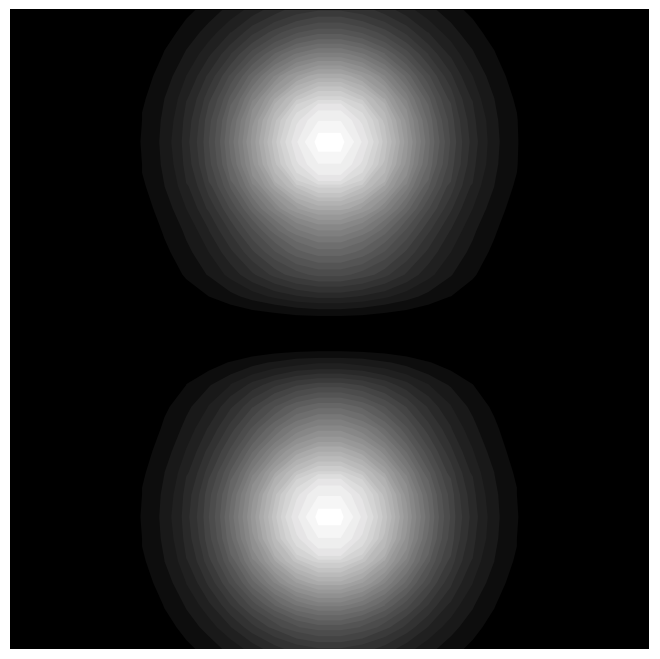

b)

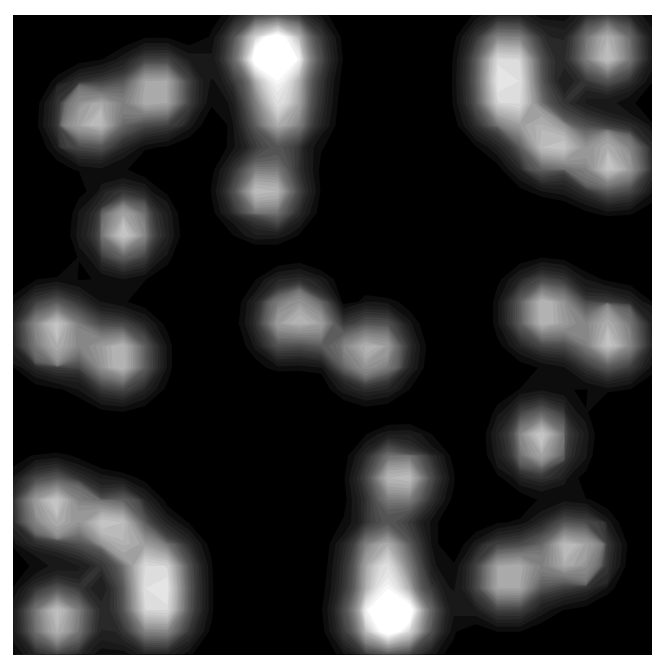

c)

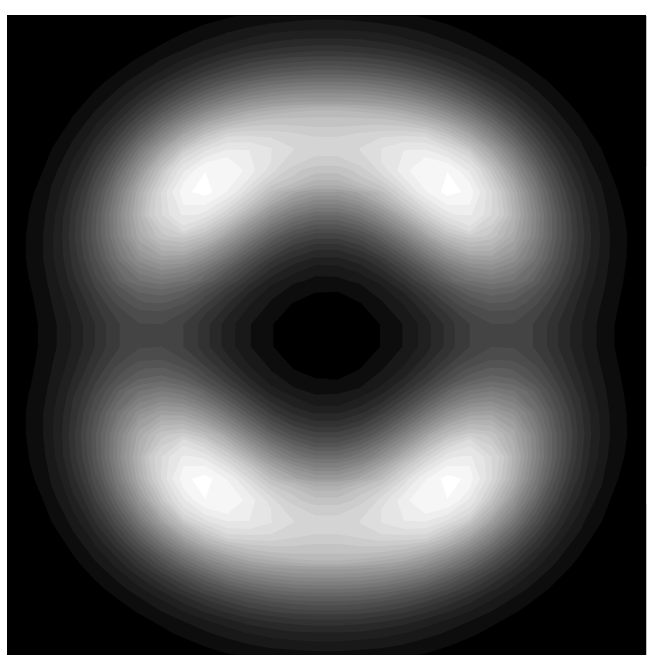

d)

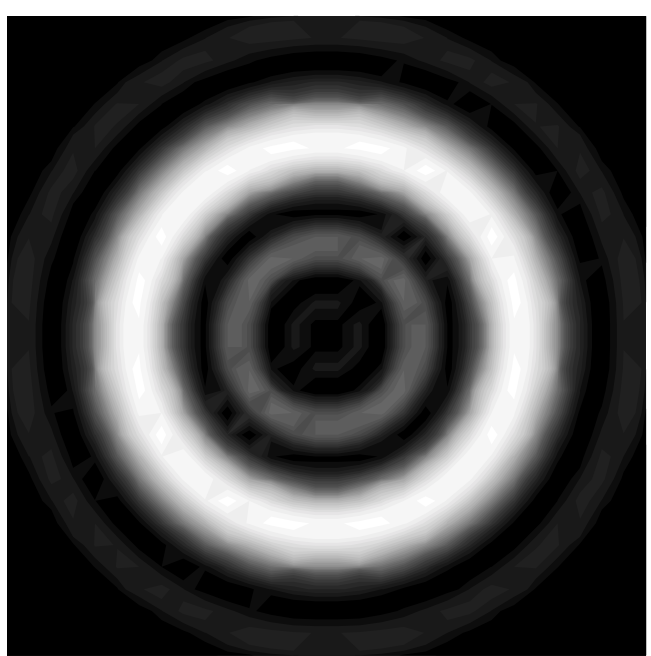

Fig. 2. The different types of the diffraction pattern for the inverse scattering at fixed moment of time: a) before averaging; b) bright spots; c) arcs; d) rings.

Let a rather broad pump beam propagates along $Z$ axis in the crystal with $\mathrm{C}_{3 \mathrm{~V}}$-symmetry. Its wave vector $k_{\varepsilon}$ in spherical coordinates can be written as:

$$
\vec{k}_{\varepsilon}=k_{\varepsilon}\left(\sin \theta_{\omega} \cos \varphi_{\omega}, \sin \theta_{\omega} \sin \varphi_{\omega}, \cos \theta_{\omega}\right), \theta_{\omega}<<1 .
$$

In the paraxial approximation, we obtain the following expression:

$$
\vec{e}_{p}=\left(\sin \psi_{\omega}, \cos \psi_{\omega},-\theta_{\omega}\left(\varphi_{\omega}-\psi_{\omega}\right)\right), \quad \psi_{\omega} \in(0,2 \pi)
$$

where the $\psi_{\omega}$ determines the angle of polarization rotation. When taking into account the symmetry properties the Glass vector at small angles $\theta_{\omega}$ has the form:

$$
\begin{aligned}
& \vec{G}=\left(-\beta_{22} \sin 2 \psi_{\omega}-\beta_{15} \theta_{\omega} \cos \psi_{\omega} \cos \left(\varphi_{\omega}-\right.\right. \\
& \left.\left.-\psi_{\omega}\right),-\beta_{22} \cos 2 \psi_{\omega}-\beta_{15} \theta_{\omega} \sin \psi_{\omega} \cos \left(\varphi_{\omega}-\psi_{\omega}\right), \beta_{31}\right) \approx \\
& \left.\approx\left(-\beta_{22} \sin 2 \psi_{\omega},-\beta_{22} \cos 2 \psi_{\omega}\right), \beta_{31}\right) .
\end{aligned}
$$

After introducing the unit vector of rotation $e_{f}=\left(\sin 2 \psi_{\omega}\right.$, $\left.\cos 2 \psi_{\omega}\right)$, the latter expression can be rewritten as:

$$
\vec{G}=\vec{G}_{\perp}+G_{z} \vec{e}_{z}, \quad \vec{G}_{\perp}=\beta_{22} \vec{e}_{f}, \quad G_{z}=\beta_{31} \vec{e}_{z}
$$

Then the Glass vector also rotates in XY-plane due to the polarization rotation, and its direction is defined by $e_{f}$.

Allowing for $\vec{\theta}_{f} \sim \vec{q}_{f \perp} \sim \vec{G}_{\perp}$, one obtains that $\vec{q}_{f \perp}=q_{f \perp} \vec{e}_{f}, \quad \vec{\theta}_{f}=\theta_{f} \vec{e}_{f}$. Hereinafter we restrict ourselves by the consideration of ergodic processes. Thus, instead of summing the result over the polarization, i.e. over the $e_{f}$ directions, we should average the result over the statistical assemble with the distribution function defined from definite models.

For the «bad» samples we should take into account the fluctuations of impurity concentrations. These variations causes the qualitative changes of crystal symmetry, due to the fact that defects «try to concentrate near the transverse growth non-homogeneous», which originates near the doped atoms and «grow through» the sample in 


\section{A. N. Morozovska et al.: Dynamics of photoinduced instability in ferroelectric...}

$z$ direction. In such a way the crystal symmetry undergoes local variations. In order to describe similar effects by our theory, we assumed that the «bad» sample can be divided into rather great amount of small regions or so called «blocks». Each block has its own $e_{f}$ direction and impurity concentration $n_{d}^{0}$, i.e. the value of $\theta_{f}$ and $\Omega_{f}$. Therefore in general case, the expression (7) has to be averaged both over $\Omega_{f}$ and over the value and the direction of the vector $\theta_{f}$. The way of averaging or summing depends on the accepted model of block distribution and sizes. Let us discuss the following model.

1) The model of «rather good» sample, or «thick» crystal without significant growth defects. The concentration $n_{d}^{0}$ can be regarded constant in all parts of the sample, and pump polarization vector $e_{p}$ makes sufficient amount of turns in the crystal due to photoinduced delay of the extraordinary beam. Thus we can average only over $e_{f}$ directions of vector $\theta_{f}$, all its directions being equiprobable, i.e. (7) can be averaged over $e_{f}$ directions with uniform distribution function for all possible angles (from 0 to $2 \pi$ ) of $e_{f}$ rotation. As the result the scattering indicatrix acquires axial symmetry, because two «spots» of side maxima transform into the ring at $\theta^{2}=\theta_{f}^{2}$ on a screen (see Fig. 2d). The dynamics of such rings is considered in detail in the next item. For «rather good» samples the PILS is periodical.

2) The model of «not rather good» sample, or «thick» crystal with pronounced block structure. If this structure is arbitrary, the size of blocks and the values of aforementioned parameters for each block can vary significantly. We can imagine so small or «microscopic» blocks with random parameters that the pump polarization vector fails to rotate at any detectable angle when pump wave passing through it. The existence of such blocks in the real sample will lead to the appearance of flickering bright spots randomly situated on the screen (see Fig. 2b). Besides, rather big or «macroscopic» blocks will rotate the pump polarization vector at different significant angles. For each «macroscopic» block one can apply the results of the previous model, taking into account that the distribution function for $e_{f}$ directions will be uniform for different angle intervals. The result of such averaging will be flickering (each with its own intensity, frequency and radius) arcs or irregular (each with its own intensity, thickness and eccentricity in different points) rings on the screen (see Fig. 2c). For such samples the PILS is quasi-periodical. If the amount of blocks tends to infinity and their parameters vary continuously, the summing over them must be substituted by integration with defined distribution function of material parameters. In particular under integration over frequency $\Omega_{f}$, (see our previous paper [11] for comparison), we obtain that one ring or several arcs of scattering light once appear and disappear on the screen (see item 3.5). For such samples the PILS is aperiodical.

Let us discuss the polarization of the direct and inverse waves after transmission of the crystal. In crystals belonged to $\mathrm{C}_{3 \mathrm{~V}}$ symmetry group the value of the photoinduced delay of the extraordinary beam $\Delta$, propagat- ing at small angle to the optical axes, significantly dominates (starting even from a small pump intensity) over the same value without illumination and is equal to: $\Delta=k_{0 z} \chi_{22}\left(E_{f y}-E_{f x}\right) n_{0}^{3}$ [16]. Thus, with changing the sign of the projection $k_{0 z}$, the direction of polarization rotation also changes. In other words, the crystal is lefthanded or right-handed active medium in dependrncy on the direction of light propagation. If light is passing through the sample «there and back», all polarization changes will be compensated. Thus, we can conclude that if a linearly polarized wave incidents on the sample along optical axes, the polarization of the direct wave in general will be close to the elliptic one, and the polarization of the inverse wave reflected from the back plane will coincide with the incident pump wave one.

The averaging in accordance with the model (1) permits set forth below simple analytical calculation. As the result we obtained rather a simple and obvious description of the optical autowaves generation. The summing in accordance with the model (2) for quasi-periodical PILS was performed numerically (see Fig. 2). For the proposed initial system and non-homogeneities model it is much simpler to obtain the analytical calculation for the aperiodical PILS or the dynamic halo scattering then for the system considered in our previous work [11].

\subsection{Autowave scattering in PRC}

Let us perform the averaging of scattering amplitude (7) over the directions of unit vector $e_{f}$ which defines the direction of $\theta_{f} \sim q_{f} \sim l_{g \perp} \sim G_{\perp}$. We supposed that all $e_{f}$ directions are equiprobable, i.e. the distribution function is uniform.

Let us introduce the following definitions:

$$
\begin{aligned}
& \vec{\theta}=\theta \vec{e}_{\theta}, \quad \vec{\theta}_{f}=\theta_{f} \vec{e}_{f}, \quad \theta=\sqrt{\theta_{x}^{2}+\theta_{y}^{2}}, \\
& \theta_{f}=\sqrt{\theta_{f x}^{2}+\theta_{f y}^{2}}, \quad\left(\vec{\theta} \vec{\theta}_{f}\right)=\theta \theta_{f} \cos \varphi,
\end{aligned}
$$

Taking into account that angle $\varphi \in[-\pi, \pi]$, one can integrate over $\varphi$ and derive the approximate expression for all argument values by means of the integral representation and asymptotic expansion for the Bessel function of zero order $J_{0}[20]$, i.e.:

$$
\frac{1}{2 \pi} \int_{-\pi}^{\pi} d \varphi \exp \left(-\eta^{2}\left(1+i n_{f}^{ \pm}\right) \theta \theta_{f} \cos \varphi\right)=J_{0}\left(\eta^{2}\left(n_{f}^{ \pm}-i\right) \theta \theta_{f}\right),
$$

$$
J_{0}\left(\eta^{2}\left(n_{f}^{ \pm}-i\right) \theta \theta_{f}\right) \sim \frac{\exp \left(\eta^{2}\left(1+i n_{f}^{ \pm}\right) \theta \theta_{f}\right)}{\sqrt{1+\eta^{2}\left(1+i n_{f}^{ \pm}\right) \theta \theta_{f}}}
$$

Thus the averaging of scattering amplitude (7) over angle $\varphi$ reduced to the using of formulas $(12,13 \mathrm{a})$. Having done this we obtain from (9): 


$$
\begin{aligned}
& \left\langle j_{1}(\theta, \tau)\right\rangle=\exp \left(-\eta^{2} \theta^{2}\right) \times \\
& \times\left|\exp \left(-i n_{f} \eta^{2} \theta^{2} / 2\right)+\sum_{ \pm} R_{1}^{ \pm} J_{0}\left(\eta^{2}\left(n_{f 1}^{ \pm}-i\right) \theta \theta_{f}\right) \exp \left( \pm i\left(\tau+\varphi_{f}\right)-\eta^{2}\left(1+i n_{f 1}^{ \pm}\right) \theta_{f}^{2} / 2-i n_{f 1}^{ \pm} \eta^{2} \theta^{2} / 2\right)\right|^{2}, \\
& \left\langle j_{2}(\theta, \tau)\right\rangle=R^{2} \exp \left(-\eta^{2}\left(\theta^{2}+\theta_{f}^{2}\right) \mid \begin{array}{l}
J_{0}\left(\eta^{2}\left(n_{f 2}^{+}-i\right) \theta \theta_{f}\right) \exp \left(2 i\left(\tau+\varphi_{f}\right)-i \eta^{2}\left(n_{f 2}^{+}-n_{f 2}^{-}\right)\left(\theta^{2}+\theta_{f}^{2}\right) / 2\right)+\left.\right|^{2} \\
+J_{0}\left(\eta^{2}\left(n_{f 2}^{-}-i\right) \theta \theta_{f}\right)
\end{array}\right.
\end{aligned}
$$

In (14b) we assume that for the inverse scattering $\left|R_{2}{ }_{2}\right| \approx\left|R^{-}{ }_{2}\right|=R$. The profiles of the averaged indicatrix (14) look like periodical running rings on a screen, which are well visible in the inverse geometry of the experiment (see Fig. 3a), where the central maximum is absent, and hardly detectable on the background of the bright center in direct geometry (see Fig. 3b).

The simple for analysis approximate expression for the inverse scattering indicatrix can be derived from (13b) and (14b):

$$
\begin{aligned}
& \left\langle j_{2}(\theta, \tau)\right\rangle \approx R^{2} \frac{\exp \left(-\eta^{2}\left(\theta-\theta_{f}\right)^{2}\right)}{1+\eta^{2} \theta \theta_{f}} \times \\
& \times \cos ^{2}\left(\varphi_{f}+\tau-\eta^{2}\left(n_{f 2}^{+}-n_{f}^{-}\right)\left(\theta-\theta_{f}\right)^{2} / 4\right) .
\end{aligned}
$$

It is clear from the analysis of (15a) and numerical simulation of the precise formula (14) that for the «rather good» samples the inverse scattering is periodical in time and posses conic spatial structure with the cone opening oscillating around the angle $\theta_{f}$. The amount, the velocity and the direction of motion of rings on the screen both for direct and inverse scattering are determined by the value and the sign of the difference $\Delta n_{f}=n_{f 1,2}^{+}-n_{f 1,2}^{-}$. At $\left(\eta \theta_{f}\right)^{2}>>1$ we can distinguish the two types of rings: the more intensive or «bright» and the less intensive or «pale», that have different directions of motion (see below the comments to (16)). For positive $\Delta n_{f}$, «bright» rings move from the angle $\theta_{f}$ to the center $\theta=0$, and "pale» ones strongly damping travel from the angle $\theta_{f}$ to the greater angles; for negative $\Delta n_{f}$ «bright» rings move from the center to the angle $\theta_{f}$, and «pale» ones travel from the circumference to the angle $\theta_{f}$, increasing their intensity and finally merge with the «bright» rings. The velocity of motion and the amount of rings increase with the increasing $\Delta n_{f}$ absolute value: at small $\left|\Delta n_{f}\right|$ only the one slowly moving «bright» ring appears, and at big $\left|\Delta n_{f}\right|$ several fast moving rings can be observable in both directions simultaneously.

As it follows from the sluggishness of photorefractive medium, the intensity of scattering must be negligibly small in the initial moment of laser switching $t=0$. In a)

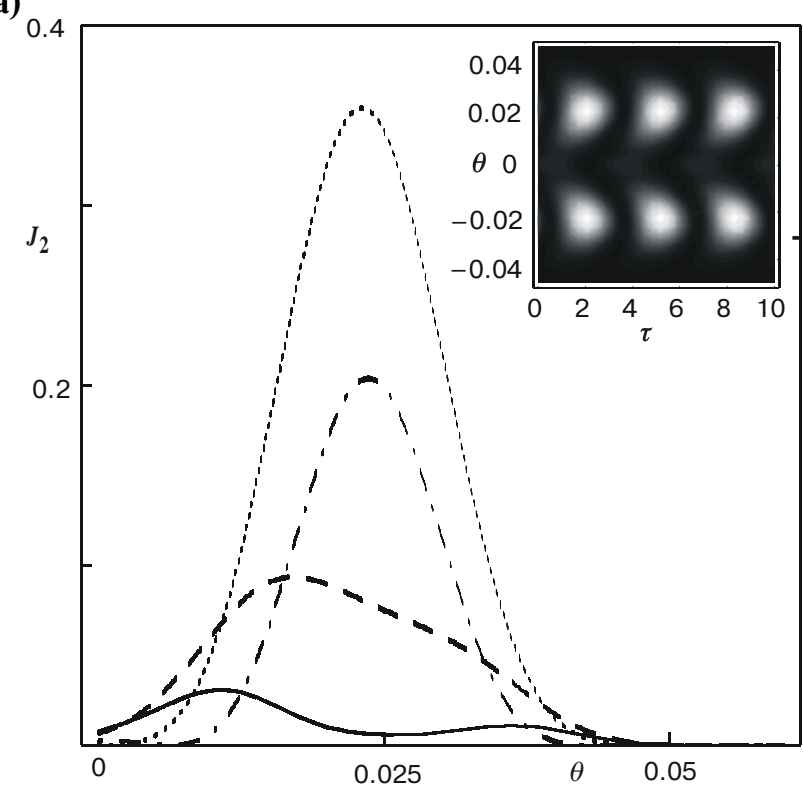

b)

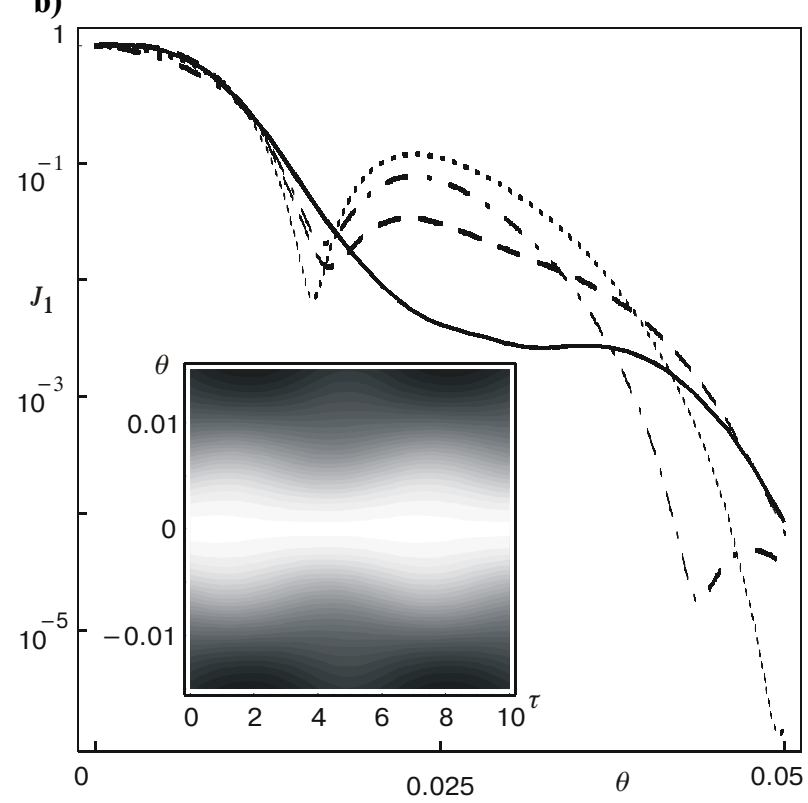

Fig. 3. The profiles of the averaged indicatrix of the inverse (a) and direct (b) autowave scattering (14) at different $\tau: \tau=\pi / 2$ (solid curves), $5 \pi / 8$ (dashed curves), $\pi$ (dotted curves), $6 \pi / 5$ (dash-dotted curves). The parameters $\eta=100, \theta_{f}=0.025 \mathrm{rad}, R^{+}{ }_{1}=R^{-}{ }_{1}=R^{+}{ }_{2}=R^{-}{ }_{2}=0.1$, $n_{f}=1$ and $\Delta n_{f}=-1$. The dynamics of autowaves in variables time-angle is shown in the insets. 


\section{A. N. Morozovska et al.: Dynamics of photoinduced instability in ferroelectric...}

this paper we have considered the stationary dynamic regime and have not taken into account the damping relaxational processes which provide the fulfillment of the real initial conditions. Therefore, the parameter $\varphi_{f}$ determining the phase of the inner field oscillations at $t=0$ remains undetermined. Having noted that at $\varphi_{f}=p / 2$ the scattering intensity $(14,15 \mathrm{a})$ is minimum at $t=0$, and at $\left(\eta \theta_{f}\right)^{2}>>1 \mathrm{it}$, besides this, is exponentially small at the initial moment, we can possibly apply our results $(14,15 a)$ for small times at

$$
\varphi_{f}=\pi / 2
$$

If the inequality $0 \leq 4(\tau-\pi(m+1 / 2)) / \Delta n_{f} \leq 1(m=0$; $1 ; 2 \ldots)$ is satisfied, one derives the approximate dependence of the angular ring size $\theta_{r}$ over $t$ from (15), having equated cosines to \pm 1 . Thus, the following dependence is obtained:

$$
\begin{aligned}
& \theta_{r}^{\mp}=\theta_{f} \mp \frac{2}{\eta} \sqrt{\frac{\tau-\pi(m+1 / 2)}{n_{f 2}^{+}-n_{f 2}^{-}}}, \\
& n_{f 2}^{+}-n_{f 2}^{-}>0 \rightarrow \tau-\pi(m+1 / 2)>0, \\
& n_{f 2}^{+}-n_{f 2}^{-}<0 \rightarrow \tau-\pi(m+1 / 2)<0 .
\end{aligned}
$$

Where sign «-» corresponds to «bright» rings, and «+» to «pale» ones, the number $\mathrm{m}$ is chosen in accordance with (16b). Really, as it follows from (15a), always $j_{2}\left(\theta_{r}^{-}\right)>j_{2}\left(\theta_{r}^{+}\right)$, and if $\Delta n_{f}<<1$ and $\left(\eta \theta_{f}\right)^{2}>>1$ than $j_{2}\left(\theta_{r}^{-}\right)>>j_{\mathrm{I}}\left(\theta^{+}{ }_{r}\right)$.

As the final remark to this item, we would like to emphasize the following. Our theory predicts that high quality ferroelectric photorefractive ilmenites, in the case when photoactive impurity does not destroy their initial structure, can generate optical autowaves even under stationary laser irradiation.

\subsection{The discussion and analyses of the tempo- ral and spatial characteristics of the scattered irradiation. Comparison with the experiment on autowave PILS in $\mathrm{LiNbO}_{3}: \mathrm{Fe}$.}

Notice that theoretically calculated diffraction pattern for inverse scattering, at least for the «thick» crystal without essential growth defects (i.e. $l>>l_{\mathrm{c}}$ and $\left|q^{\prime \prime}{ }_{z} l\right|>>1$ ), possesses significantly higher visualization, than the same for the direct scattering (see Fig. 3). That is why in this item, we restrict ourselves by calculation and analysis of those basic characteristics of inverse scattering, which have been compared with the available experimental data on autowave scattering in $\mathrm{LiNbO}_{3}$ crystals, doped with $0.02 ? 0.07$ wgh. $\%$ Fe revealed before [6] and intensively studied now in our lab.

Let us calculate such an important characteristic as the spatial correlation coefficient of the inverse scattering, hereinafter designated as $K_{a b}$. Namely let us derive the correlation function of the two points «a» and «b»» on the screen (characterized by angles $\theta_{a}$ and $q_{b}$ ) with different (in general) time-averaged intensities $j_{2}\left(\vec{\theta}_{a}\right)=$ $=\left\langle\left\langle j_{2}\left(\vec{\theta}_{a}, \tau\right)\right\rangle\right\rangle_{\tau}$ and $j_{2}\left(\vec{\theta}_{b}\right)=\left\langle\left\langle j_{2}\left(\vec{\theta}_{b}, \tau\right)\right\rangle_{\tau}\right.$. By definition $K_{a b}=\frac{2 j_{2}\left(\vec{\theta}_{a}\right) j_{2}\left(\vec{\theta}_{b}\right)}{\left(j_{2}\left(\vec{\theta}_{a}\right)\right)^{2}+\left(j_{2}\left(\vec{\theta}_{b}\right)\right)^{2}}$.

In fact, we calculate $K_{a b}$ for two different «flickering spots» or parts of «flickering arcs» or points of «irregular rings». Within the framework of the proposed models of nonhomogeneities, the conic cross-sections with various vectors $\theta_{f a}, \theta_{f b}$ and amplitudes $R_{a}, R_{b}$ can be realized in the points «a» and «b». After time-averaging in (9) and (14) with different $\theta_{f a}$ and $\theta_{f b}$ one can obtain after using (6) that in the case when side maxima are good resolved (i.e. $\left(h q_{f}\right)^{2}>>1$ ), the result in the form:

$$
\begin{aligned}
& \frac{j_{2}\left(\vec{\theta}_{b}\right)}{j_{2}\left(\vec{\theta}_{a}\right)}=K_{0} \exp \left(-\eta^{2}\left(\left(\vec{\theta}_{b}-\vec{\theta}_{f b}\right)^{2}-\left(\vec{\theta}_{a}-\vec{\theta}_{f a}\right)^{2}\right)\right), \\
& K_{0} \sim \frac{R_{b}^{2}}{R_{a}^{2}} \frac{1+w^{2} \theta_{f a}^{4}}{1+w^{2} \theta_{f b}^{4}} .
\end{aligned}
$$

Here $R_{a, b}$ and $w$ do not depend on the angles $\theta_{f a}$ and $\theta_{f b}$. For the case, when

$\theta_{a} \approx \theta_{b} \approx \theta_{f a} \approx \theta_{f b}$,

(18) can be essentially simplified and rewritten as the function of dimensionless intensity $\xi$ introduced in item 2 . In accordance with the numerical calculations (see Fig. 1b) for the good compensated ferroelectric-semiconductor $(h \geq 0.9)$ even at rather small pump intensities (as soon as $s I_{0} \gg s_{0}$ ) the Eq. (18a) from item 2 for $q_{f \perp}$ is valid, i.e. $q_{f \perp} \sim \xi^{-1 / 2}$. Using Eq. (18a) from item 2 and (19), we obtain $\theta_{a} \approx \theta_{b} \approx \theta_{f a} \approx \theta_{f b}=\theta_{0} / \sqrt{\xi}$. Then $K_{a b}$ reaches the form:

$$
\begin{aligned}
& K_{a b}(\xi)=\frac{2 K_{0} \exp \left(-\xi_{0} / \xi\right)}{1+K_{0}^{2} \exp \left(-2 \xi_{0} / \xi\right)}, \\
& \xi=\left(s I_{0}+s_{0}\right) / m_{d}^{0}, \\
& \xi_{0} \approx \eta^{2}\left(\cos \left(\angle \vec{\theta}_{b}, \vec{\theta}_{f b}\right)-\cos \left(\angle \vec{\theta}_{a}, \vec{\theta}_{f a}\right)\right)
\end{aligned}
$$

Hereinafter in this item, we assume that it is possible to neglect the dark conductivity due to sufficient laser intensity $s I_{0} \gg s_{0}$, i.e. to substitute

$s_{0}=0$.

Then, in order to obtain the dependence of $K_{a b}$ on the pump intensity $I_{0}$, it is sufficient to make the substitution $\xi / \xi_{0} \rightarrow I_{0} / I_{0 \mathrm{~K}}$ in (20). In the case, the values $I_{0 K}$ and $K_{0}$ are fitting parameters. The dependence of the spatial correlation coefficient $K_{a b}$ over $\xi / \xi_{0}$ and comparison with recently obtained experimental data are represented in Fig. 4. While comparing experimental data with the dependence $K_{a b}$ on $I_{0}$ we used (20), and therefore conside- 


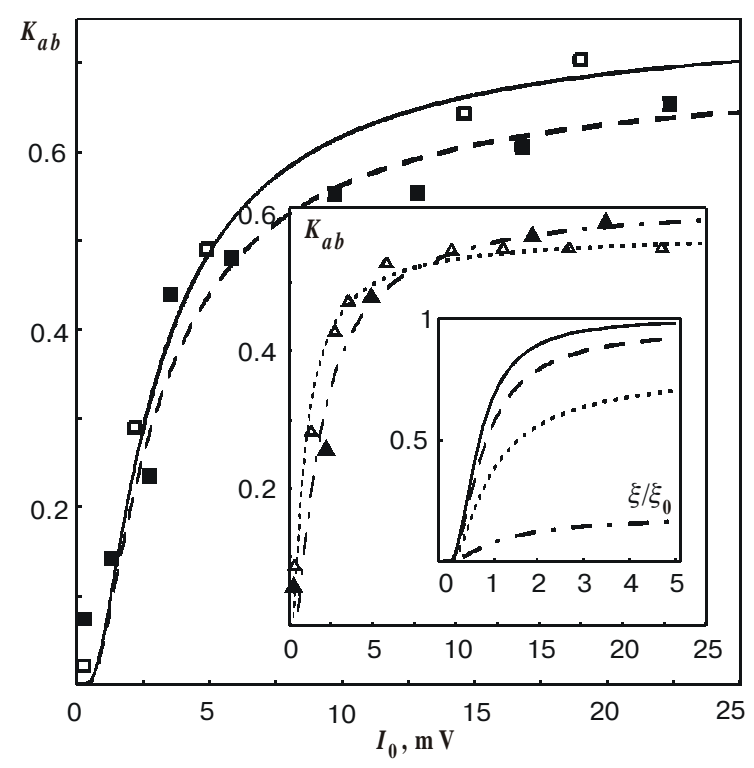

Fig. 4. The dependence of the measured and calculated spatial correlation coefficient $\mathrm{K}_{\mathrm{ab}}$ over intensity $I_{0}$ at the different angles between the detectors: $10^{\circ}$ (empty squares and solid curve at $K_{0}=0.46, I_{0 \mathrm{~K}}=2.86 \mathrm{~mW}$ ), $30^{\circ}$ (filled squares and dashed curve at $K_{0}=0.41, I_{0 \mathrm{~K}}=2.86 \mathrm{~mW}$ ), $90^{\circ}$ (empty triangles and dotted curve at $K_{0}=0.32, I_{0 \mathrm{~K}}=0.83 \mathrm{~mW}$ ), $180^{\circ}$ (filled triangles and dash-dotted curve at $K_{0}=0.35, I_{0 \mathrm{~K}}=1.6 \mathrm{~mW}$ ). The dependence of $K_{\mathrm{ab}}$ over $\xi / \xi_{0}$ at different $\mathrm{K}_{0}$ is depicted in the inset: $K_{0}=0.1$ (dash-dotted curve); 0.5 (dotted curve); 0.8 (dashed curve); 1 (solid curve).

red the register detectors placed on the ring in accordance with (19) and assumed that they were the point ones (i.e. we approximate the real apparatus function by the Dirac delta-function). The latter approximation is pertinent only if $\theta_{d} \ll<\left|\vec{\theta}_{b}-\vec{\theta}_{a}\right|\left(\theta_{d}\right.$ is the angular detector size).

We'd like to remind that inverse scattering has the conic spatial structure with the cone opening oscillating around $\theta_{f}($ see (15)). It is appeared convenient to characterize these temporal oscillations by the minimum angle $\theta_{\min }$ (i.e. the angle of cone generation), the angle of the best visualization $\theta_{0}$ (i.e. the angle of cone maximum intensity), and the maximum angle $\theta_{\max }$ (i.e. the angle of cone disappearance). The experimentally measured values of $\theta_{\max }$ and $\theta_{\min }$ can be additionally limited by the resolution of the register hardware. We determined these angles numerically from (14). It is turned out that for a wide region of parameters $\eta$ и $\Delta n_{f}$, with high accuracy one can apply clear and simple relations:

$$
\begin{aligned}
& \theta_{0} \approx\left(\theta_{f \max }+\theta_{f \min }\right) / 2 \approx \theta_{f}, \\
& \theta_{f \min } \approx f_{1} \theta_{f}, \quad \theta_{f \max } \approx f_{2} \theta_{f} .
\end{aligned}
$$

Here $f_{1}<1, f_{2}>1$ are positive constants. Thus, the cone parameters are determined by the angle $q_{f}$, which increases with the increasing photoactive impurity concentration $n_{d}^{0}$, because $q_{f} \sim n_{d}^{0}$. Using Eq. (15a) from item 2, (9) or (14-15) and (21) we obtain:

$$
\begin{aligned}
& \theta_{f}=\theta_{g} \frac{1}{h} \sqrt{\frac{f(h)-1}{f^{3}(h)}}, \quad f(h)=\frac{1-p+\sqrt{(1-p)^{2}+4 p / h}}{2}, \\
& h=n_{a} / n_{d}^{0}, \quad h<1, \quad p=m_{a} / s I_{0},
\end{aligned}
$$

where $\theta_{g}$ does not depend on $h$. Calculated in accordance with $(14-15,22-23)$ dependences of the angles $\theta_{\min }, \theta_{0}$ and $\theta_{\max }$ over impurity concentration $n_{d}^{0}$ have been compared with the experimentally obtained ones in Fig. 5.

Let us discuss the temporal spectrum of scattering waves. The theoretical (see Eq. (17) from item 2) and experimental dependences of autowave frequency $\mathrm{W}_{f}$ over pump intensity $I_{0}$ are presented in Fig. 5. To all appearances, we possess the experimental data for the linear part of the dependence Eq. (17) from item 2, i.e. for well compensated samples $(h \approx 1)$ or/and small $I_{0}$ (see Fig. 1a and Eq. (18a) from item 2). Within the framework of our theory for «thick crystals» the autowave frequency does not depend on the crystal thickness. Possibly that this dependence is present below the thickness threshold at $\left|q_{f z}^{\prime \prime} l\right| \leq 1$. The temporal dependence of the autowaves intensity averaged over ring can be easily calculated from (15). In comparing the result with the experimentally obtained autowaves evolution (see inset in Fig. 6), to our

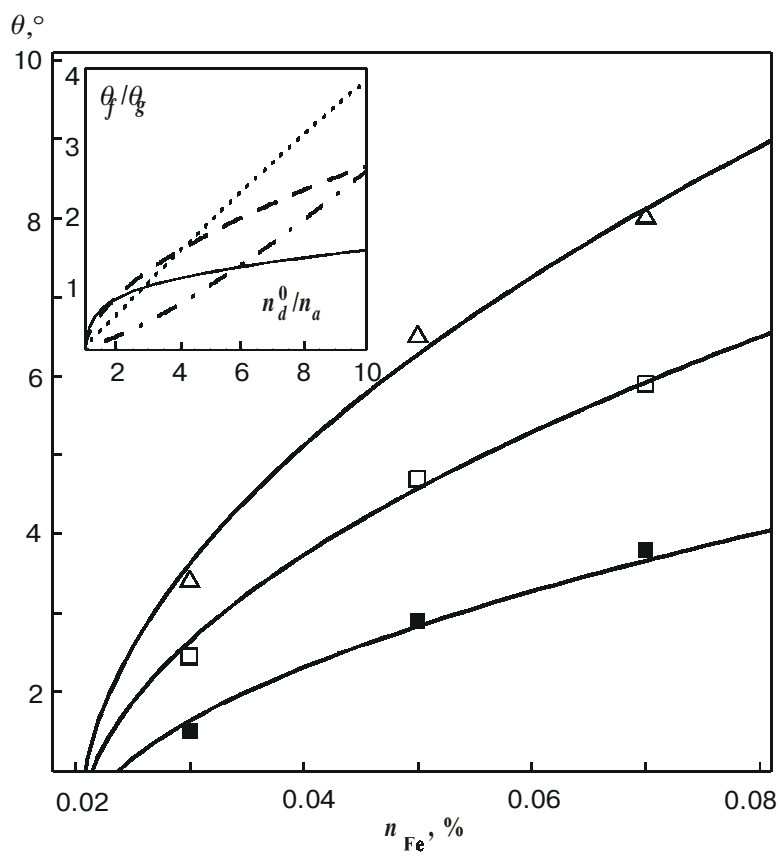

Fig. 5. The dependence of $\theta_{\min }$ (filled squares), $\theta_{0}$ (empty squares), $\theta_{\max }$ (empty triangles) over Fe concentration in $\mathrm{LiNbO}_{3}$. The best agreement with the experiment was obtained at $p=1.5$, $n_{a}=0.02, \theta_{g}=4.85^{\circ}, f_{1}=0.62, f_{2}=1.37$. The dependence of the ratio $\theta_{f} / \theta_{g}$ over $n_{d}^{0} / n_{a}$ at different $\mathrm{p}$ are shown in the inset: $p=0.01$ (dash-dotted curve), 0.1 (dotted curve), 1 (dashed curve), 10 (solid curve). 


\section{A. N. Morozovska et al.: Dynamics of photoinduced instability in ferroelectric...}

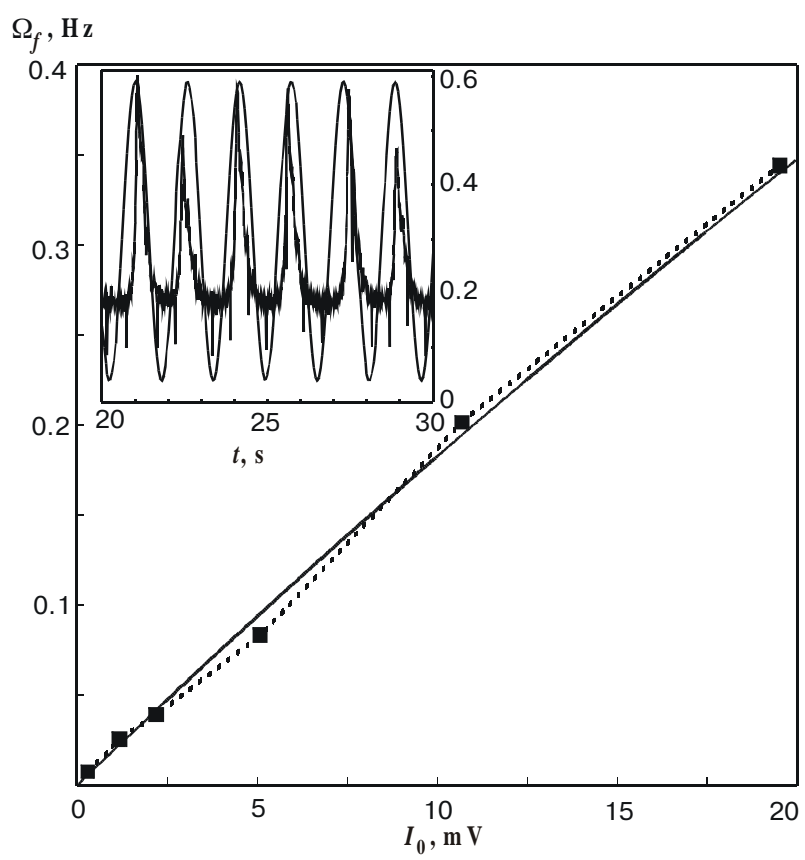

Fig. 6. Theoretical (solid curve, calculated by Eq.(17) from item 2 at $h=0.95, \gamma n^{0}{ }_{d}=8.2 \mathrm{~Hz}$ ) and experimental (filled squares) dependencies of autowaves frequency $\Omega_{f}$ over $I_{0}$. Inset: the experimental (bold curve) and theoretical (fine curve) dependences of the averaged over the ring autowave intensity (in arbitrary units) over time.

mind the qualitatively compliance in general dynamics of the process is evident, the differences of the experimental and theoretical profiles on the period is related with the linearity of the theory. In fact we omitted all nonlinear terms in the initial system, i.e. neglected the possibility of the crystal to generate sub- and super-harmonics.

The most simple for the analysis and the best corresponded with the experiment is the dynamics of the rings of autowave scattering at small $\Delta n_{f}$ (see Fig. 7). In this case rather bright ring appears on a screen, then extends with decreasing of its thickness and gradually disappears. After this the crystal generates the following ring.

Note, that autowave PILS was found only in the photoinduced bleaching regime. All the dynamic effect disappears in the reflecting regime, i.e. under turning the sample over $180^{\circ}$ in XY-plane. This fact confirms the validity of the corresponding speculations from the item 3.1.

Also the refractive index of the outer medium does not influence on the existence of autowaves: the crystal generates them both in the air and in the immersion or even in the water. This confirms the assumption made in the item 3.1 about the fact that the seeding for the inverse wave is exactly internal reflection at the boundary of the screening region and the double electric layer.

The conclusion of the item 3.2 about the reconstruction of pump wave polarization for the inverse scattered waves and its destruction for the direct ones has found the experimental confirmation with a high accuracy up to angular seconds.

In our lab all the above-mentioned dynamic effects (see Fig. 2) have been registered only in the high quality $\mathrm{LiNbO}_{3}$ single crystals. Similar effects have not been found in any other samples. In accordance with the conclusion of the item 3.3, we explain these results by the presence of the non-zero component $G_{\perp}$ only in the ilmenite $\mathrm{LiNbO}_{3}$ and its absence in all other investigated samples.

Thus, though the real temporal spectrum of PILS has been approximated by delta-function in our linearized theory, the main spatial and temporal characteristics of the process obtained in this way are in a full agreement with the available experimental data.
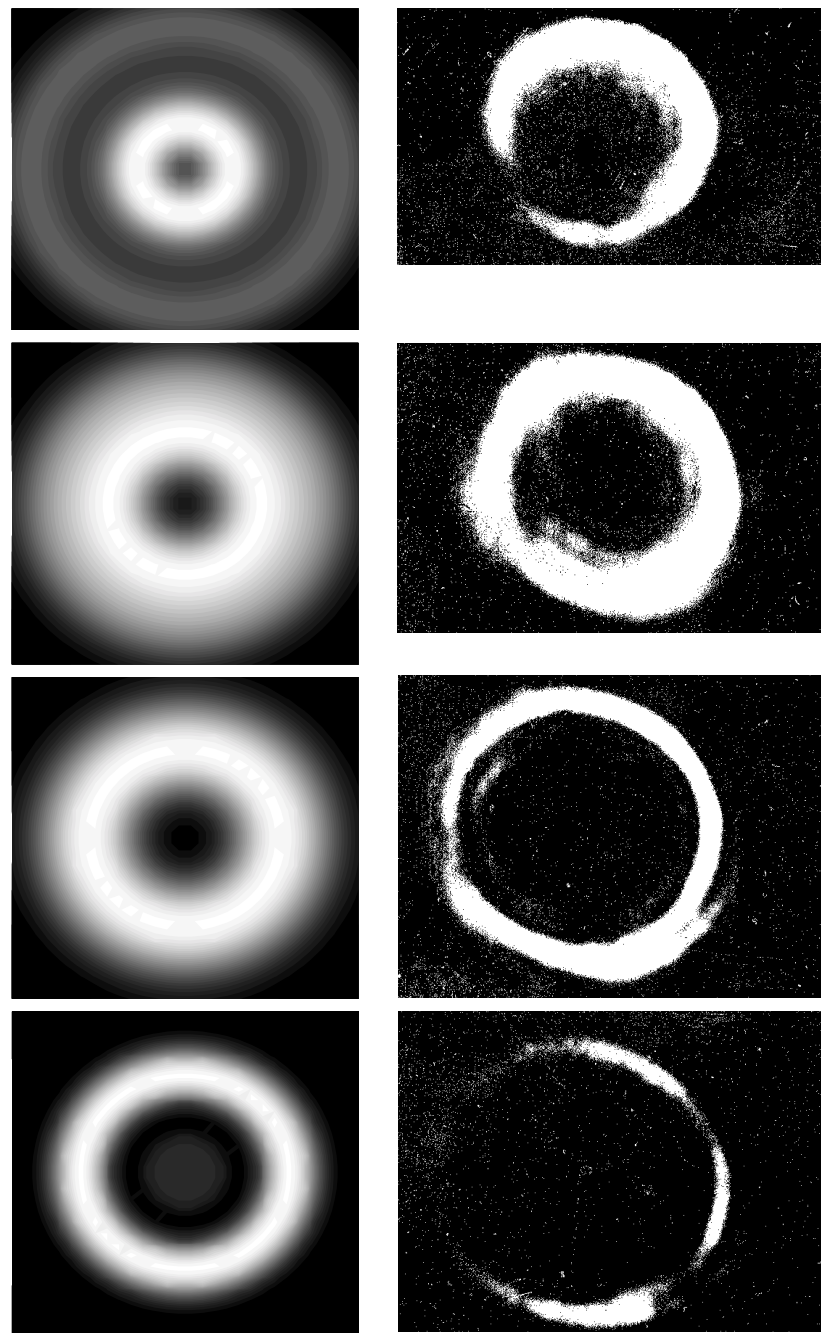

Fig. 7. The typical dynamics of the autowave scattering rings in the successive moments of time: appearance, spreading out and disappearance. Left side is the theoretical calculation by $(14 \mathrm{~b}$, $15 \mathrm{~b})$ at $\eta=100, \theta_{f}=0.025 \mathrm{rad}, R^{+}{ }_{2}=R^{-}{ }_{2}=0.1, \Delta n_{f}=-1$ and $\tau=\pi / 3$, $2 \pi / 3, \pi, 7 \pi / 6$ (from top to bottom). Right side is the photographic image of the ring evolution on a screen. 


\section{A. N. Morozovska et al.: Dynamics of photoinduced instability in ferroelectric...}

\subsection{Dynamic halo scattering in PRC. Compari- son with the experiment on non-stationary isotropic PILS in $\mathrm{LiTaO}_{3}: \mathrm{Cr}$.}

Let us consider the dynamic PILS in the «thick» crystal with developed system of growth defects. In order to calculate the scattering indicatrix for such samples besides averaging of (9) over polarization, it is necessary to average it over the other material parameters such as absolute value of $G_{\perp}$, impurity $n_{d}^{0}$ and acceptors $n_{a}$ concentrations, etc. Within the framework of our model, the latter can be reduced to averaging over the absolute value of angle $\theta_{f} \sim G_{\perp}$ and the frequency $\Omega_{f}$, which can be substituted by integration in the case when fluctuating values changes quasi-continuously. Actually, we have supposed that the regions with different material parameters are so numerous, that summing over them can be substituted by statistical averaging of (14) over variables $\theta_{f}$ and $\Omega_{f}$ with definite continuous joint distribution function $P\left(\theta_{f}, \Omega_{f}\right)$. Also we assume that deviations of $\theta_{f}$ and $\mathrm{W}_{f}$ from their mean values $\theta_{f 0}$ and $\Omega_{f 0}$ are small and independent. Therefore, one can postulate that the distribution $P\left(q_{f}, \mathrm{~W}_{f}\right)$ has Gaussian form:

$$
\begin{aligned}
& P\left(\theta_{f}, \Omega_{f}\right)= \\
& =\frac{\exp \left(-\left(\theta_{f}-\theta_{f 0}\right)^{2} / 2 \Delta_{\theta}^{2}-\left(\Omega_{f}-\Omega_{f 0}\right)^{2} / 2 \Delta_{\Omega}^{2}\right)}{\pi \Delta_{\theta} \Delta_{\Omega}}, \\
& \theta_{f 0} \gg \Delta_{\theta}, \quad \Omega_{f 0} \gg>\Delta_{\Omega} .
\end{aligned}
$$

Using (9), (136) and (24), the integration limits over $q_{f}$ and $\mathrm{W}_{f}$ can be extended and the averaging of indicatrix (14) can be rewritten as:

$$
\begin{aligned}
& \left\langle j_{1,2}(\theta, t)\right\rangle=\left|\int_{-\infty}^{\infty} d \Omega_{f} d \theta_{f} P\left(\theta_{f}, \Omega_{f}\right) j_{1,2}(\theta, \tau)\right|^{2}, \\
& j_{1,2}(\theta, t) \approx R_{1,2}^{0} \exp \left(-\eta^{2}\left(1+i n_{f}\right) \theta^{2} / 2\right)_{+} \\
& +\sum_{ \pm} R_{1,2}^{ \pm} \frac{\exp \left( \pm i \Omega_{f} t-\eta^{2}\left(1+i n_{f 1,2}^{ \pm}\right)\left(\theta-\theta_{f}\right)^{2} / 2\right)}{\sqrt{1+\eta^{2}\left(1+i n_{f 1,2}^{ \pm}\right) \theta \theta_{f}}},
\end{aligned}
$$

$R_{1}^{0}=1, \quad R_{2}^{0}=0$.

In (25) the integration over frequency can be performed precisely, and that over the angle can be appreciated by the Laplace method [21]. Finally we derived:
As it follows from (26), at $t>>1 / \Delta_{\Omega}$ 《side» components of the fields are damped as $\exp \left(-\left(t \Delta_{\Omega}\right)^{2} / 2\right)$. Eventually after the averaging over the continuous frequency spectrum the periodical function transforms in to the aperiodical one. The averaging over the angle $\theta_{f}$ does not cause new effects, it only re-determines the dispersion $h$. Therefore, for the simplification of the further analysis, we assume that

$\left(\Delta_{\theta} \eta\right)^{2}<<1$.

The inequality (27) make it possible to average field amplitudes over frequency spectrum exactly, i.e. without approximation (13b). Thus, instead of (26) we have:

$$
\left\langle j_{1,2}(\theta, \tau)\right\rangle=\left|\begin{array}{l}
R_{1,2}^{0} \exp \left(-\eta^{2}\left(1+i n_{f}\right) \theta^{2} / 2\right)+ \\
+\sum_{ \pm}^{ \pm} R_{1,2}^{ \pm} J_{0}\left(\eta^{2}\left(n_{f 1}^{ \pm}-i\right) \theta \theta_{f 0}\right) \exp \left(-\Delta_{\Omega}^{2} t^{2} / 2 \pm\right. \\
\left. \pm i \Omega_{f 0} t-\eta^{2}\left(1+i n_{f 1,2}^{ \pm}\right)\left(\theta^{2}+\theta_{f 0}^{2}\right) / 2\right)
\end{array}\right|
$$

Taking into account (13b), (15b) and (27), the scattering indicatrix (28) can be reduced to:

$$
\begin{aligned}
& \left\langle j_{1}(\theta, t)\right\rangle \approx|\times| \begin{array}{l}
\exp \left(-\eta^{2}\left(1+i n_{f}\right) \theta^{2} / 2\right)+ \\
+R \cdot \exp \left(-\Delta_{\Omega}^{2} t^{2} / 2-\eta^{2}\left(\theta-\theta_{f 0}\right)^{2} / 2\right) \times \\
\left(\frac{\exp \left(i \Omega_{f 0} t-\eta^{2} i n_{f 1}^{+}\left(\theta-\theta_{f 0}\right)^{2} / 2\right)}{\sqrt{1+\eta^{2}\left(1+i n_{f 1}^{+}\right) \theta \theta_{f 0}}}-\right. \\
\frac{\exp \left(-i \Omega_{f 0} t-\eta^{2} i n_{f 1}^{-}\left(\theta-\theta_{f 0}\right)^{2} / 2\right)}{\sqrt{1+\eta^{2}\left(1+i n_{f 1}\right) \theta \theta_{f 0}}}
\end{array} \mid
\end{aligned} \mid,
$$

It follows from (28-29) that the obtained PILS indicatrix differs from $(14,15)$ only by the presence of damping factors for the «side» components. Then the analysis of the spatial structure of the periodical PILS,

$$
\left\langle j_{1,2}(\theta, \tau)\right\rangle=\left|\begin{array}{l}
R_{1,2}^{0} \exp \left(-\eta^{2}\left(1+i n_{f}\right) \theta^{2} / 2\right)+ \\
+\sum_{ \pm} R_{1,2}^{ \pm} \frac{\exp \left(-\Delta_{\Omega}^{2} t^{2} / 2 \pm i \Omega_{f 0} t-\eta^{2}\left(1+i n_{f 1,2}^{ \pm}\right)\left(\theta-\theta_{f 0}\right)^{2} / 2\left(1+\Delta_{\theta}^{2} \eta^{2}\left(1+i n_{f 1,2}^{ \pm}\right)\right.\right.}{\sqrt{1+\eta^{2}\left(1+i n_{f 1,2}^{ \pm}\right) \theta \theta_{f 0}}}
\end{array}\right|^{2}
$$




\section{A. N. Morozovska et al.: Dynamics of photoinduced instability in ferroelectric...}

performed in the item 3.3, remains valid for the aperiodical one with the correction that in latter case one can see only the finite number or rings with the consequently decreasing intensity on the screen (see Fig. 8).

Let us compare the obtained results with the experiment on the non-stationary isotropic PILS in $\mathrm{LiTaO}_{3}: \mathrm{Cr}$ [5]. Above all, we would like to notice that despite another geometry of the experiment (pump beam was directed not along $Z_{C}$, but along crystallographic axis $X_{C}$ ), our results can be applied after the appropriate re-designation of tensors components. Surely, this operation changes the numerical values of some parameters, but maintains the analytical structure of formulae (26-29).

The authors of [5] observed on a screen a ring of PILS (we called it «halo») once appeared and gradually dis-
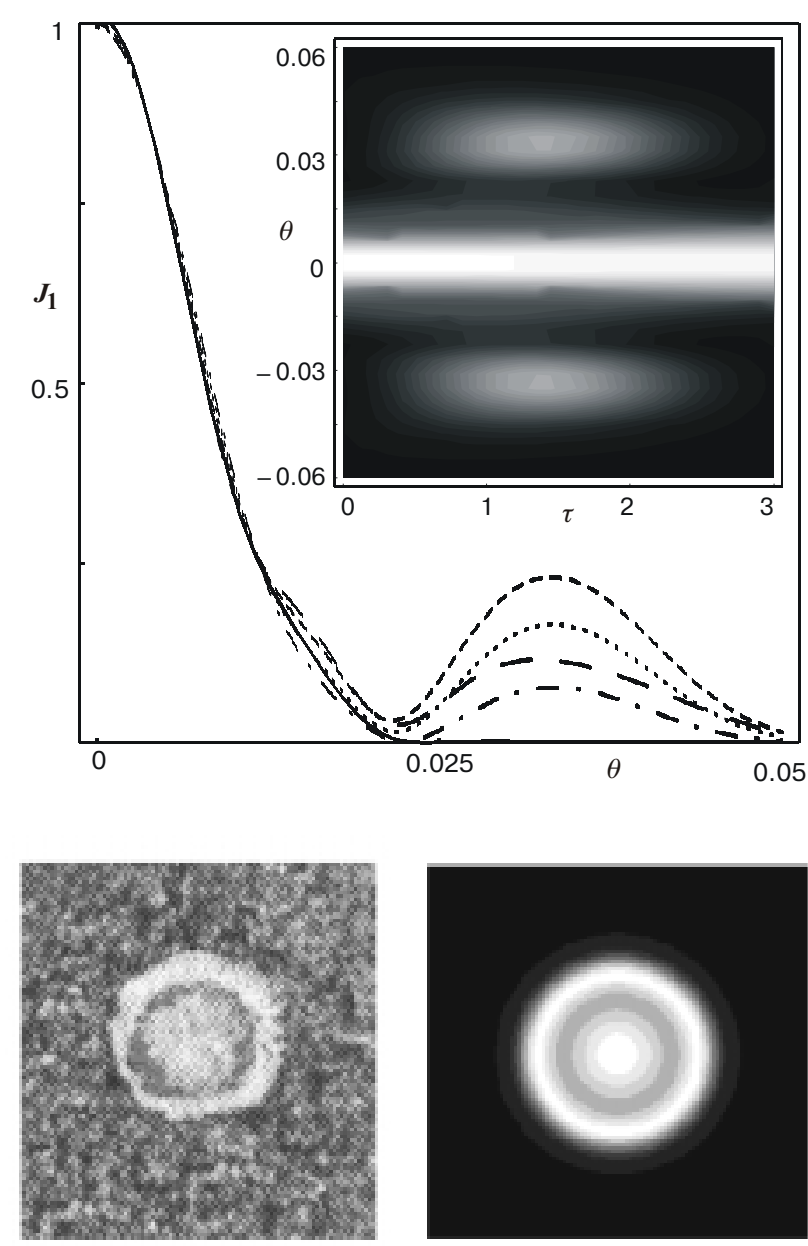

Fig. 8. The profiles of the averaged indicatrix of the direct aperiodical scattering (29) at different $\tau$ : $\tau=0$ (solid curves), $\pi / 5$ (longdashed curves), $\pi / 2$ (short-dotted curves), $5 \pi / 8$ (dotted curves), $3 \pi / 4$ (dash-dotted curves). The parameters $\eta=100, R=1, n_{f}=1$ and $\Delta n_{f}=-1 \theta_{f}=0.035 \mathrm{rad}$. The dynamics of halo in variables time-angle is shown in the inset. The halo structure on a screen are depicted below. Left side is the photographic image of the halo on a screen [5]. Right side is the theoretical calculation by (29) at $\eta=100, \theta_{f}=0.035 \mathrm{rad}, R=1, \Delta n_{f}=-1$ and $\tau=\pi / 3$. appeared around the laser pump beam passing through the sample. They have not reported about any similar effects for the inverse scattering. Within the framework of the proposed theory, this phenomenon can be explained, if we assume that only the «side» components of the direct wave possess sufficient intensity, and the structure of the inverse scattering do not visualize itself due to the insignificantly small intensity. In accordance with (28), this is possible if:

$\exp \left(-\Delta_{\Omega}^{2} t_{0}^{2}\right)<<1, \quad t_{0} \sim \eta^{2}\left|n_{f 1,2}^{+}-n_{f 1,2}^{-}\right| \theta_{f 0}{ }^{2} / \Omega_{f 0}$,

where $t_{0}$ is the moment of the ring appearance.

The exact expression for $t_{0}$ depends on the properties of the registering detectors. In that case, the scattering is absent at $t<t_{0}$ and at $t>t_{0}(28)$ can be rewritten as follows:

$$
\begin{aligned}
& \left\langle j_{1}(\theta, t)\right\rangle \approx \exp \left(-\eta^{2} \theta^{2}\right)+R \cdot \exp \left(-\Delta_{\Omega}^{2} t^{2} / 2-\eta^{2} \theta^{2} / 2-\right. \\
& \left.-\eta^{2}\left(\theta-\theta_{f 0}\right)^{2} / 2\right) \times \\
& \times\left(\begin{array}{l}
\frac{\cos \left(\Omega_{f 0} t-\eta^{2} n_{f 1}^{+}\left(\theta-\theta_{f 0}\right)^{2} / 2+\eta^{2} n_{f} \theta^{2} / 2\right)}{\sqrt[4]{\left(1+\eta^{2} \theta \theta_{f 0}\right)^{2}+\eta^{4}\left(n_{f 1}^{+} \theta \theta_{f 0}\right)^{2}}}- \\
-\frac{\cos \left(-\Omega_{f 0} t-\eta^{2} n_{f 1}\left(\theta-\theta_{f 0}\right)^{2} / 2+\eta^{2} n_{f} \theta^{2} / 2\right)}{\sqrt[4]{\left(1+\eta^{2} \theta \theta_{f 0}\right)^{2}+\eta^{4}\left(n_{f 1} \theta \theta_{f 0}\right)^{2}}}
\end{array}\right)+ \\
& +O\left(\exp \left(-\Delta_{\Omega}^{2} t^{2}\right)\right), \\
& \left\langle j_{2}(\theta, \tau)\right\rangle \sim O\left(\exp \left(-\Delta_{\Omega}^{2} t^{2}\right)\right)<<\exp \left(-\Delta_{\Omega}^{2} t^{2} / 2\right) .
\end{aligned}
$$

It follows from the numerical analysis of (28-31) that at the appropriate values of the fitting parameters of the ring of direct PILS or dynamic halo-scattering (see Fig. 8) once appeared, slightly broadened and then disappeared on a screen. Thus, the results obtained in this item are in a qualitative agreement with the experiment [5].

\section{Conclusions}

In this paper we have studied consecutively the influence of the recharging trap waves on the dynamics of the inner photoinduced fields in the uniaxial ferroelectric PRC under stationary laser irradiation. The general form and the characteristic features of the direct and inverse PILS have been derived and discussed. The obtained results can be briefly formulated as following statements.

For the first time, it has been shown that, due to influence of transverse photovoltaic current and the typical boundary conditions for all components of inner field, among all possible scenarios of transverse instabilities, the boundary circle will be realized in the perfect crystal. Due to this phenomenon, the periodical, quasi-periodical or aperiodical PILS appear in the system, depending on the structure of growth in homogeneities and the character of photoinduced fluctuations caused by them. 


\section{A. N. Morozovska et al.: Dynamics of photoinduced instability in ferroelectric...}

The correlation between the structure of scattering light and the type of fluctuations has been considered in details for ilmenites. The great attention has been paid to the description of the optical autowaves generation and dynamic halo scattering in these materials.

All the main theoretical results are in a good agreement with the available experimental data and describe the phenomena found in $[5,6]$.

\section{References}

1. N.N. Rozanov, Hysteresis phenomena in distributed optical systems // JETP. 80(1), pp. 96-108 (1981).

2. J. Takacs and L. Solymar, Subharmonics in $\mathrm{Bi}_{12} \mathrm{SiO}_{20}$ with an applied ac electric field // Opt.Lett. 17(2), pp.247-248 (1992).

3. D. Engin, S. Orlov, M. Segev, G.C. Valley, A. Yariv, Orderdisorder phase transition and critical slowing down in photorefractive self-oscillators // Phys.Rev. Lett. 74(10), pp. 1743-1746 (1995).

4. I.V. Dzedolik, A.V. Volyar, The registration of optical vortices in refractive media // Proceedings of SPIE. 3904, pp. 8391 (1999).

5. Kh.S. Bagdasarov, V.B. Batoev, E.M. Uyukin, Non-stationary Isotropic Photoinduced Light Scattering in $\mathrm{LiTaO}_{3}: \mathrm{Cr} / /$ Quant.Elect. 13(10), pp. 1962-1964 (1986).

6. V.V. Lemeshko, V.V. Obukhovskii, Autowaves of photoinduced light scattering // Pis'ma.Zh.Tekh.Fiz. 11(11), pp. 13891393 (1985)

7. O. Sandfuchs, J. Leonardy, F. Kaiser and M. Belic, Transverse instabilities in photorefractive //Phys. Rev. A53(6), pp.4519 (1996).
8. T. Honda, P. Banerjee, Threshold for spontaneous pattern formation in reflection-grating-dominated photorefractive media with mirror feedback //Opt. Lett. 21(11), pp. 779-781 (1996).

9. B.I. Sturman, E. Sharmonina, M. Mann, K.H. Ringhofer, Space-charge waves in photorefractive ferroelectrics // J.Opt.Soc.Am. 12(9), pp. 1672-1650 (1995).

10. A.N.Morozovska, V.V. Obukhovskii, Autowave-type instability in photorefractive crystals // Proceedings of SPIE. 3904, pp. 209-214 (1999).

11. A.N. Morozovska, V.V.Obukhovskii, Dynamic halo-scattering in photorefractive crystals // Proceedings of SPIE. 4355, pp. 22-227 (2001).

12. A.S. Furman, Spontaneous increasing of recharging waves in crystals without inversion center under stationary illumination // Sol.St.Phys. 29(4), pp. 1076-1085 (1985).

13. N.V. Kukhtarev, V.B. Markov, S.G. Odulov, M.M. Soskin, V.L. Vinetskii, Holographic Storage in Electrooptic crystals / I Ferroelectrics, 22, pp. 949-960 (1979).

14. V.M. Fridkin, Ferroelectrics-semiconductors, M. Nauka, (1976) (in Rus).

15. G.M. Guro, I.I. Ivanchik, N.F. Kovtonuk, C-domain crystal of barium titanate in shorted capacitor // Sol.St.Phys. 11(7), pp. ф1956-1964 (1969).

16. U.S. Kuzminov, Litium niobate and tantalate are the materials for nonlinear optics, M. Nauka (1975) (in Rus).

17. A.N. Tikhonov, A.A. Samarskii, The equation of mathematical physics, M. Nauka, pp. 510-521 (1972), (in Rus.)

18. A. Yariv, P. Yeh, Optical waves in crystals, N.-Y. (1985).

19. M. Born, E. Wolf, The principles of optics, M.: Nauka (1973) (in Rus).

20. M. Abramowitz and A. Stegun, Handbook of Matematical Functions, Dover Publications, INC. N.Y., frm.9.2.1 (1979).

21. A.G. Sveshnikov, A.N. Tikhonov, Theory of functions of complex variables, M. Nauka, pp.253-260 (1970) (in Rus). 